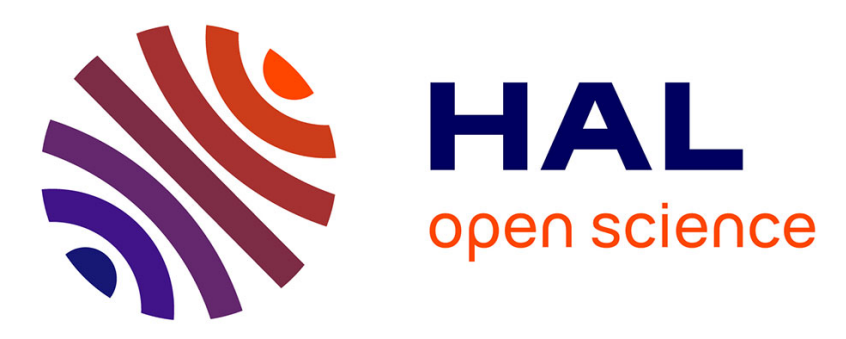

\title{
Roughness signature of tribological contact calculated by a new method of peaks curvature radius estimation on fractal surfaces
}

Maxence Bigerelle, Jean-Marie Nianga, Denis Najjar, Alain Iost, Cédric Hubert, K. J. Kubiak

\section{To cite this version:}

Maxence Bigerelle, Jean-Marie Nianga, Denis Najjar, Alain Iost, Cédric Hubert, et al.. Roughness signature of tribological contact calculated by a new method of peaks curvature radius estimation on fractal surfaces. Tribology International, 2013, 65 (65), pp.235-247. 10.1016/j.triboint.2013.03.017 . hal-01065878

\section{HAL Id: hal-01065878 https://hal.science/hal-01065878}

Submitted on 4 Oct 2017

HAL is a multi-disciplinary open access archive for the deposit and dissemination of scientific research documents, whether they are published or not. The documents may come from teaching and research institutions in France or abroad, or from public or private research centers.
L'archive ouverte pluridisciplinaire HAL, est destinée au dépôt et à la diffusion de documents scientifiques de niveau recherche, publiés ou non, émanant des établissements d'enseignement et de recherche français ou étrangers, des laboratoires publics ou privés. 


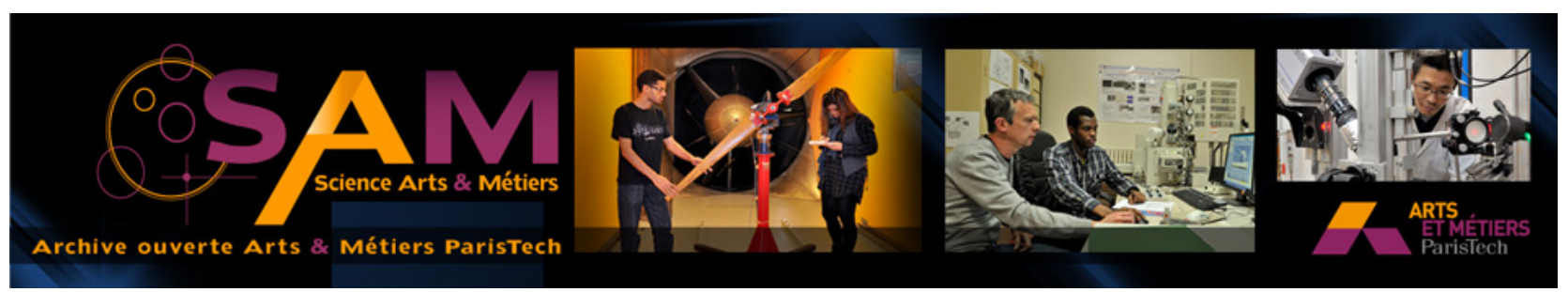

\section{Science Arts \& Métiers (SAM)}

is an open access repository that collects the work of Arts et Métiers ParisTech researchers and makes it freely available over the web where possible.

This is an author-deposited version published in: http://sam.ensam.eu

Handle ID: .http://hdl.handle.net/10985/8561

\section{To cite this version :}

M. BIGERELLE, J. M. NIANGA, D. NAJJAR, Alain IOST, C. HUBERT, K. J. KUBIAK - Roughness signature of tribological contact calculated by a new method of peaks curvature radius estimation on fractal surfaces - Tribology International n65, p.235-247 - 2013 


\title{
Roughness signature of tribological contact calculated by a new method of peaks curvature radius estimation on fractal surfaces
}

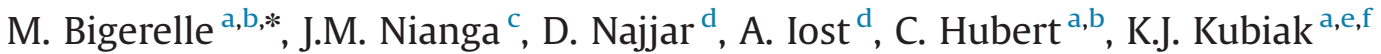 \\ a Université de Valenciennes, Laboratoires TEMPO/ LAMIH, 59313 Valenciennes Cedex 9, France \\ ${ }^{\mathrm{b}}$ PRES Lille Nord de France, Lille Cedex, France \\ ' Equipe Mécanique des Structures, HEI, 13 Rue de Toul, 59046 Lille Cedex, France \\ d LML, UMR CNRS 8107, F-59650 Villeneuve d'Ascq, Arts et Métiers ParisTech, France \\ e University of Leeds, School of Mechanical Engineering (iETSI), Leeds LS2 9JT, United Kingdom \\ ${ }^{\mathrm{f}}$ University of Liverpool, School of Engineering, Liverpool L69 3GH, United Kingdom
}

Keywords:

Roughness

Friction

Curvature radius

Fractal

\begin{abstract}
A B S T R A C T
This paper proposes a new method of roughness peaks curvature radii calculation and its application to tribological contact analysis as characteristic signature of tribological contact. This method is introduced via the classical approach of the calculation of radius of asperity. In fact, the proposed approach provides a generalization to fractal profiles of the Nowicki's method [Nowicki. Wear Vol. 102, p. 161-176, 1985] by introducing a fractal concept of curvature radii of surfaces, depending on the observation scale and also numerically depending on horizontal lines intercepted by the studied profile. It is then established the increasing of the dispersion of the measures of that lines with that of the corresponding radii and the dependence of calculated radii on the fractal dimension of the studied curve. Consequently, the notion of peak is mathematically reformulated. The efficiency of the proposed method was tested via simulations of fractal curves such as those described by Brownian motions. A new fractal function allowing the modelling of a large number of physical phenomena was also introduced, and one of the great applications developed in this paper consists in detecting the scale on which the measurement system introduces a smoothing artifact on the data measurement. New methodology is applied to analysis of tribological contact in metal forming process.
\end{abstract}

\section{Introduction}

In general, the physical responses are due to interactions between physical processes and some surface characteristic parameters, such as the geometrical ones, and in particular, the curvature radius from which an estimate could be easily obtained for periodic or stochastic surfaces. However, although its importance is very often underestimated, this one appears in the mathematical formulation of numerous physical models: in optics, it represents a threshold, under which the reflected beam on a surface could not be modelled by the Kirchhoff method, in tribology, it plays an important role in the determination of the contact pressure.

Generally, the calculation of the radius of curvature requires rather smooth curves of studied surfaces, however it is not always the case when dealing with fractal surfaces, as it was shown in
Mandelbrot's works [1,2]. Furthermore, as all the metric parameters relative to a fractal curve depend on the scale of measurement, it thus becomes particularly difficult to give a sense to the notion of local radius of curvature for fractal surfaces. In tribological contact fractal surface are often used to avoid sensibility to scale of measurement [3-5]. However, some methods thanks to Fourier analysis were proposed with the aim of the estimation of the radius of curvature [6,7]. Let us note moreover that, for special classes of surfaces, for which the spectrum could be related to the fractal dimension, the curvature radius could be estimated. However, restrictive conditions of surfaces, as the self-affinity, as well as the existence of artifacts in the Fast Fourier Transform, make that method uncertain. Furthermore, the representation of the radius of curvature in the Fourier space has not been extensively studied. Numerous questions arise then: What really the radius of curvature for a fractal curve means? Does it possess a geometrical meaning? What is the interest of its eventual estimation? What could we deduct, from physical models, based on the consideration of such a parameter? All these questions show the necessity to give a geometrical formulation of the curvature radius for a fractal curve. Consequently, in this paper, we suggest 
establishing, at the same time, the dependence of the radius of curvature on the scale under which the studied surface is observed, as well as, its relation to the fractal dimension of that surface. So, as the properties of the fractal curve are defined from the fractal dimension, the regular non fractal surface is then influenced by the fractal dimension of the studied real surface. Consequently, even under a formulation supposing regular surfaces, as the physical formulation evoked above, we could not neglect the fractal dimension. To calculate this last one, there are various numerical methods, still not giving the same result, when they are applied to a modelled surface with known fractal dimension. Due to that inconsistency, the method which we should choose is the one presenting the same properties as those of the curves used in the physical model. So, if we want to estimate the influence of the fractal dimension of a surface on the estimation of the radius of curvature, we have to calculate this one, from the last one, with the same scale used for the computation.

This paper is divided into two parts:

In Part I we first review classical methods of calculation of the radius of curvature, and in particular, that of Nowicki's [8] relative to the regular curves, and for which, we proceed to an adaptation, before its extension to fractal curves. Then, we introduce a new calculation approach for the fractal dimension of surfaces. Its accuracy is tested on fractal curves with known fractal dimension, and some mathematical properties of the radius of curvature are stated.

In Part II the proposed method is applied to analysis of tribological contact in metal forming process. Variation of peaks curvature radius before and after the process is revealing detailed topographical signature of different parts of tribological contact. Therefore, the history or contact conditions can be analysed and different zones inside the contact area can be distinguished. We also show that proposed method could be coupled with an inverse methodology to obtain simulated profiles presenting the same morphology as experimental curves measured by tactical profilometer on surfaces obtained by polishing. Next presented application is an analysis of artifacts introduced by radius of tip during measurement of surface by a stylus profiler.

\section{Part I-Mathematical model of curvature radius of a rough surface}

\subsection{Model of curvature radius}

\subsection{A fractal definition of the curvature radii of a surface}

Let $\Gamma$ be the profile of a given rough surface. $\Gamma$ can be considered as the graph of a continuous function $z$, defined by

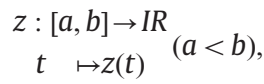

and parameterized by the real variable $t$, where the parameterization is introduced by the function $\gamma$,defined as follows:

$$
\begin{aligned}
\gamma: I R \rightarrow \Pi \\
\quad t \mapsto \gamma(t)=(t, z(t))
\end{aligned}
$$

$(t, z(t))$ represents any point of the real plane $\Pi$. The curvature $\chi(t)$ of the parameterized curve led by $\gamma$, is then defined by:

$\chi(t)=\frac{\left|z^{\prime \prime}(t)\right|}{\left|1+z^{\prime 2}(t)\right|^{3 / 2}}$
Therefore, the curvature radius $r(t)$ of the profile $\Gamma$ at the location $\mathrm{t}$, can be written such as:

$r(t)=\frac{\left(1+z^{\prime 2}(t)\right)^{3 / 2}}{\left|z^{\prime \prime}(t)\right|}$

with $\left|z^{\prime \prime}(t)\right| \neq 0$ for any $t \in[a, b]$.Consequently, the mean curvature radius of that profile, on the interval $[a, b]$, is then given by:

$\bar{r}=\frac{1}{b-a} \int_{t \in[a, b]} r(t) d t$

The derivative functions in Eqs. (3) and (4) are generally estimated by the finite differences method, which is far from being stable. Whitehouse [9] then proposed a better estimation, by using the polynomial interpolation. However, it was established, without using the fractal concept, therefore this method cannot be used [10]. Other methods based on the Fourier analysis [11] could be used, but they present the weakness not to be consistent in numerical calculation. For those reasons, Longuet-Higgins [12] proposed a statistical method based, respectively, on the distributions of the maxima and crossings of the mean level, for a random surface, but this method supposes the curvature to be statistically independent of the scanning scale. Whitehouse and Archard [13] then proposed a method using the autocorrelation function to estimate the curvature statistics. That method was subsequently modified by Sales and Thomas, who used the truncated autocorrelation function, according to the Maclaurin series expansions [14]. Moalic et al. [10] proposed the application of the finite differences method on the modified autocorrelation function, in order to estimate the repartition of the curvature of the profile. However, the authors found that errors increase with increasing of the wave number. Using now the fractal approach, Ganti and Bhushan [15] found that the curvature of the profile follows a power-law $\chi(t) \propto f_{h}^{\Delta}$, where $f_{h}$ is the Nyquist frequency of the surface, related to the resolution of the instrument, and where the parameter $\Delta$ is its fractal dimension. This theory supposes that the spectrum of the surface follows a power law $P(f) \propto f^{2 \Delta-5}$, with $f_{h}>>1 / L$, and where $L$ is the scanning length. However, as noticed by Gallant et al. [16] in the context of the estimation of the spectrum, the effect of the smoothing due to the measurement, provides yet another factor which limits the size of the frequency $f_{h}$. However, the condition $P(f) \propto f^{2 \Delta-5}$ restricts strongly the use of this method, as we could establish it, in the case of a white noise, for which $P(f)$ is constant.

The common point of all these methods is that they are based on statistical, differential or fractal properties, which could be indirectly related to the radius of curvature of the studied profile.

However, contrary to the previous ones, the method proposed by Nowicki [8] allows the study of the surface roughness, by introducing a parameter, directly measured from the surface: the so-called, radius of asperity (Fig. 1), defined as follows:

$r_{c}=l_{x}^{2} / 8 l_{y}$

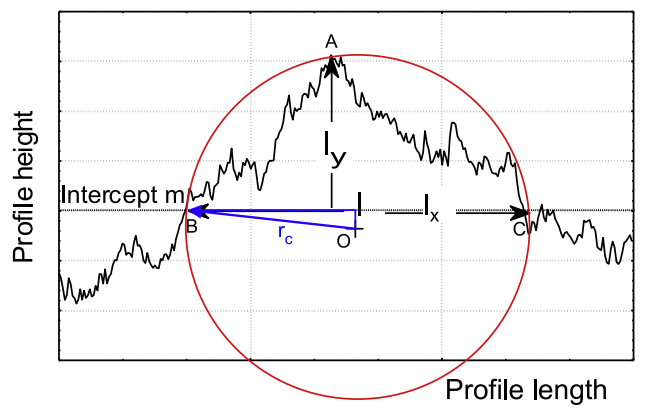

Fig. 1. Definition of $l_{x}$ and $l_{y}$ used to calculate the local curvature radius $r_{c}\left(l_{x}\right)$. 
with $l_{y}=0.1 R_{\max }$ or $l_{y} \approx 0.05 R_{\max }$, and where $R_{\max }$ is the maximal range amplitude of the profile. This method consists in finding the radius $r_{c}$ of a circle of center $\mathrm{O}$, passing by a crest $\mathrm{A}$, and by two other points $\mathrm{B}$ and $\mathrm{C}$ of the profile; these two last ones being distant of $l_{x}$. The distances which separate the line $(B, C)$, respectively, from $A$ and from $O$, are $l_{y}$ and $r_{c}-l_{y}$ The parameter $l_{y}$ is considered sufficiently small, so that the segment $[A, O]$ is supposed to be perpendicular to $[\mathrm{B}, \mathrm{C}]$, in its middle I. If we now apply Pythagoras' theorem to the triangle OBI, it then follows:

$\left(l_{x} / 2\right)^{2}+\left(r_{c}-l_{y}\right)^{2}=r_{c}^{2}$

Consequently, we get:

$r_{c}=\frac{l_{y}}{2}+\frac{l_{x}^{2}}{8 l_{y}}$

Eq. (8) is obtained, assuming that $l_{y}$ is sufficiently small, and the following condition is satisfied:

$l_{y}<l_{x}$

Nevertheless, some remarks can be drawn for such a method:

(I) The techniques to detect the peaks are not well defined. So, when $l_{y}$ value is fixed, Nowicki's method determines all local peaks (in a discretized case, if $z_{i-1}<z_{i}$ and $z_{i}>z_{i+1}$, then $z_{i}$ corresponds to a peak). In what precedes, it is assumed a uniform partition of the interval $[a, b]$, with a grid $t_{0}<\cdots<t_{i-2}<t_{i-1}<\ldots<t_{N}$, and with $z_{i} \simeq z\left(t_{i}\right), i=1,2, \ldots, N$. Then, as $l_{y}$ values are fixed, there exists for each peak, a unique value of $l_{x}$ giving the following discretized set $\left\{z_{i-q}, \ldots, z_{i-1}, z_{i}, z_{i+1}, z_{i+2}, \ldots, z_{i+p}\right\}$. The peak is retained if $z_{i-q}<\cdots<z_{i-2}<z_{i-1}<z_{i}$ and $z_{i}>z_{i+1}>z_{i+2}>\cdots>z_{i+p}$. This local radius curvature will be named Euclidian Radii Curvature with the following notation $\tilde{r}_{i}\left(t_{i}\right)$. On the other hand, the analytical method supposes that $z_{i}$ is the maximal peak of the non-discretized surface, and implies $p=q$, if the peak gets a perfect circular shape. Reciprocally, if $p \neq q$, this last one does not get such a shape.

(II) The threshold used to estimate $l_{y}=\alpha R_{\max }$ does not have any theoretical justification. Indeed, trying to determine the parameter radius of the crest, we have $l_{y}=\lim \alpha$ Rt. However, on discretized curves and for $l_{y}$ sufficiently ${ }^{\alpha} \rightarrow 0$ small, the choice of $l_{x}$ becomes indeterminate, due to the fact that $l_{x}=k \delta_{x}$, where $\delta_{x}$ is the length of the sampling interval. Furthermore, because of the stochastic aspect of the profile, the three points $\mathrm{A}, \mathrm{B}$ and $\mathrm{C}$ become more and more aligned, when the value of $l_{x}$ is decreasing, implying a dramatic increasing of the $r_{c}$ variance estimator.

(III) On experimental profiles, a smoothing effect is realized under a characteristic length [17]. For example, if the profile is recorded by a tactile profiler, the recording surface is then seen smoother at a length of the same order of magnitude than the tip curvature radius. Consequently, for $l_{y}$ sufficiently small, one has to record the curvature radius of the measurement artefacts, and the curvature radii will then be wrongly increasing.

(IV) If the surface contains some noise (white or pink), there exists a great probability for the Nowicki's algorithm to detect false peaks. Finally, the Radii of curvature get erroneous.

(V) If the profile is the result of the combination of different processes acting at various scales, the radii of curvature so obtained are different. However, it becomes evident that the detection of peaks becomes uncertain and will so favour smaller peaks.

(VI) For physical surfaces possessing a fractal aspect [18-21], the calculation of $r_{c}$ has no physical sense and the Nowicki's method will lead to different values of that parameter, depending on the sampling rate. Furthermore, it is noticed that the decreasing of the sampling rate will decrease $\tilde{r}_{c}$. This confirms that $\tilde{r}_{c}$ calculated by the Nowicki's method have no sense if we postulate that $z_{i-q}<\cdots<z_{i-2}<z_{i-1}<z_{i}$ and $z_{i}>z_{i+1}>z_{i+2}>\cdots>z_{i+p}$ (see Appendix A for more detailed justification).

\subsubsection{Theoretical relation in proposed method}

For fractal curves, $r_{c}$ depends on the scale at which the observation is made. And, as we postulate that the curvature radii could be defined at a given scale, the Nowicki's method has then to be reformulated. We will conserve the notion of $l_{x}$ without imposing any property to the points of the profile that are related to it, since $\alpha$ cannot be fixed without introducing an artefact. For these reasons, we choose to calculate $l_{x}$ by the following method:

(1) We choose a horizontal straight line at the level $h$ that crosses the profile, and we built a set of $l_{x}$ values intercepting the profile. More precisely, $z_{i}<h, \quad j \geq 1, \quad\left(z_{i+1}, z_{i+2}, \ldots, z_{i+j}\right)$ $\geq m, \quad z_{i+j+1}<m, \quad l_{x}=x_{i+j+1}-x_{i}$ where $m$ is a number of intercepting horizontal lines used in algorithm that are uniformly randomly chosen.

(2) For each $l_{x}$, the local maximal peak (maximum value of profile) is obtained which gives $l_{y}$. More precisely,

$$
\begin{gathered}
z_{i}<h, \quad j \geq 1, \quad\left(z_{i+1}, z_{i+2}, \ldots, z_{i+j}\right) \geq m, \quad z_{i+j+1}<m, \\
l_{y}=\sup _{k \in\{i+1, i+2, \cdots i+j\}}\left(z_{k}\right)-h
\end{gathered}
$$

(3) $r_{c}$ is then computed from Eq. (6), and this process is repeated for all the other elements of the set of $l_{x}$ values.

(4) Another horizontal straight line is chosen randomly and the steps 1 to 3 are repeated.

The detailed algorithm is presented in Fig. 2.

Theorem 1. If $l_{x}$ exists, for all non-constant continuous function $f$ uniformly Hölderian, anti-Hölderian, and defined on a real interval $[a, b]$, one gets, for the fractal dimension of the graph $G_{f}$ of $f$ :

$\Delta\left(G_{f}\right)=\limsup _{l_{x} \rightarrow 0}\left(\log r_{c}\left(l_{x}\right) / \log l_{x}\right)$

Rationalization of the approach: As $f$ is Hölderian in $t$, with exponent $H(0<H \leq 1)$, there exists a positive constant $c$, such that, for any $t^{\prime}$ :

$\left|f(t)-f\left(t^{\prime}\right)\right| \leq c\left|t-t^{\prime}\right|^{H}$

Eq. (11) follows Hölderian form: [22]

$v(t, \varepsilon)=\sup _{t^{\prime}, t^{\prime \prime} \in[t-\varepsilon, t+\varepsilon]}\left|f\left(t^{\prime \prime}\right)-f\left(t^{\prime}\right)\right| \leq c(t) \varepsilon^{H}$

Then if $f$ is uniformly then the constant $c$ is independent of $t$, by integration over the domain of definition $T$,

$\bar{v}(T, \varepsilon)=\int_{T} \sup _{t^{\prime}, t^{\prime \prime} \in[t-\varepsilon, t+\varepsilon]}\left|f\left(t^{\prime \prime}\right)-f\left(t^{\prime}\right)\right| d t \leq c \varepsilon^{H}$

with

$\Delta\left(G_{f}\right) \leq 2-H$

On the other hand, as $f$ is uniformly anti-Hölderian too, with the same exponent, there exists a positive constant $c^{\prime}$,independent of $t$ such that, for any $\varepsilon$ :

$\bar{v}(T, \varepsilon) \geq C^{\prime} \varepsilon^{H}$

With

$\Delta\left(G_{f}\right) \geq 2-H$ 


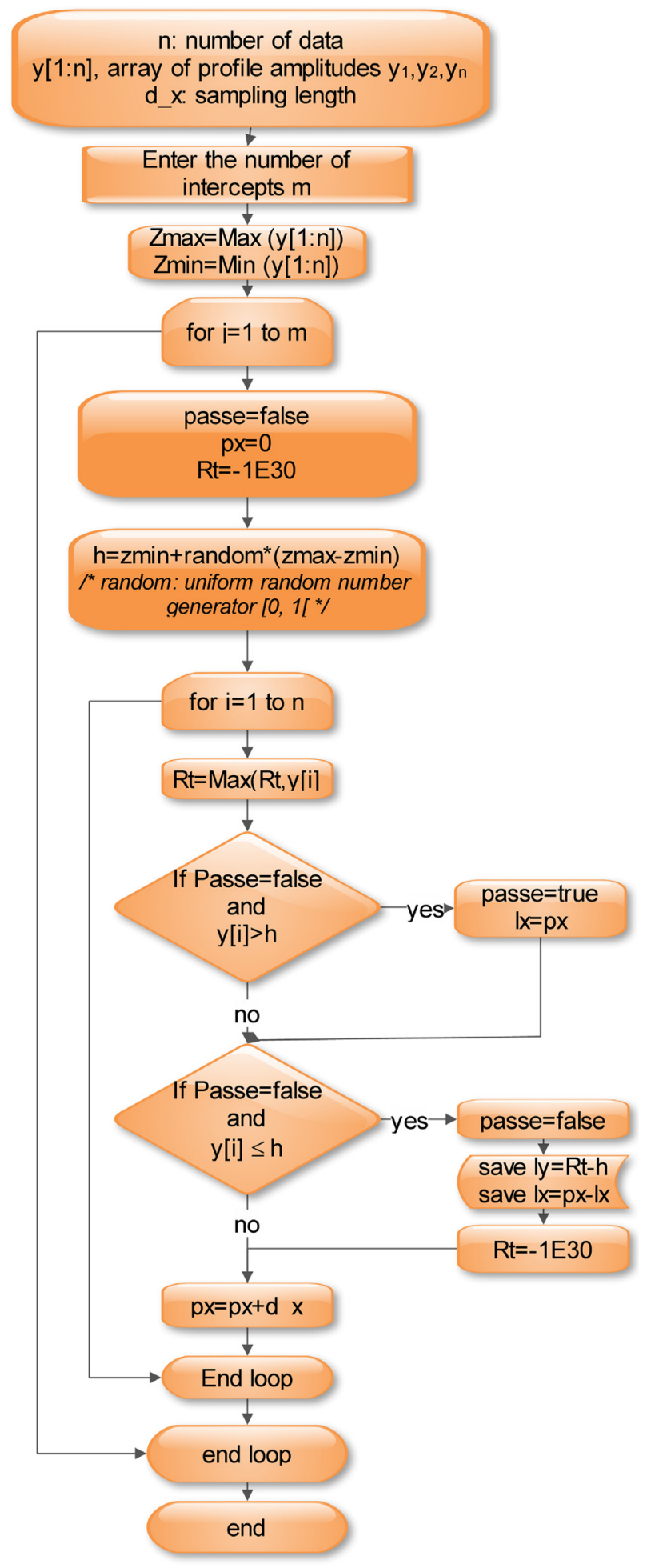

Fig. 2. Algorythm used to calculate the Curvature Radius Estimation on Fractal Surface.

Taking (12) and (14) into account, we can then write, for $\varepsilon$ taking the particular value $l_{x}(t, h) / 2$ at the level $h$ that crosses the profile (the term 1.2 is due to the fact that $v(t, \varepsilon)$ is defining on a $2 \varepsilon$ interval from Eq. (12)):

$c(t)\left(\frac{l_{x}(t, h)}{2}\right)^{H} \geq v\left(t, \frac{l_{x}(t, h)}{2}\right) \geq c^{\prime}(t)\left(\frac{l_{x}(t, h)}{2}\right)^{H}$ $\sup _{t^{\prime}, t^{\prime \prime} \in[t-\varepsilon, t+\varepsilon]}\left|f\left(t^{\prime \prime}\right)-f\left(t^{\prime}\right)\right|$

from Eq. (12) is the local range of the function and is identified with the height $l_{y}(t, h)$ of the peak of width $l_{x}(t, h)$, localized in $t$ according to our definition.

Then $v(t, \varepsilon)=l_{y}\left(\frac{l_{x}(t, h)}{2}, t\right)$ and one gets:

$c(t)\left(\frac{l_{x}(t, h)}{2}\right)^{H} \geq l_{y}\left(\frac{l_{x}(t, h)}{2}, t\right) \geq c^{\prime}(t)\left(\frac{l_{x}(t, h)}{2}\right)^{H}$

But, $f$ being uniformly Hölderian, then cand $c^{\prime}$ do not depend on $t$, and therefore [22]:

$\Delta\left(G_{f}\right)=2-H$

And summing the same $l_{x}(t, h)=l_{x}$ with $h$ values defined in $h \in\left[\inf _{t \in[T]} f(t), \sup _{t \in[T]}(t)\right]$ and $t \in[0, T]$, defining a 2D domain $\Omega\left(l_{x}\right)$ with a $A\left(\Omega\left(l_{x}\right)\right)$ area (in case of discreet set of measured profile points $A\left(\Omega\left(l_{x}\right)\right)$ is the size of this set), then:

$c \iint_{\omega \in \Omega\left(l_{x}\right)}\left(\frac{l_{x}(t, h)}{2}\right)^{H} d \omega \geq \iint_{\omega \in \Omega\left(l_{x}\right)} l_{y}\left(\frac{l_{x}(t, h)}{2}, t\right)$

$d \omega \geq C^{\prime} \iint_{\omega \in \Omega\left(l_{x}\right)}\left(\frac{l_{x}(t, h)}{2}\right)^{H} d \omega$

and from Eqs. (12) and (14)

$c A\left(\Omega\left(l_{x}\right)\right)\left(\frac{l_{x}}{2}\right)^{H} \geq \iint_{\omega \in \Omega\left(l_{x}\right)} l_{y}\left(\frac{l_{x}(t, h)}{2}, t\right) d \omega \geq c^{\prime} A\left(\Omega\left(l_{x}\right)\right)\left(\frac{l_{x}}{2}\right)^{H}$

that can be rewritten by the practical forms

$c\left(\frac{l_{x}}{2}\right)^{H} \geq \frac{1}{A\left(\Omega\left(l_{x}\right)\right)} \iint_{\omega \in \Omega\left(l_{x}\right)} l_{y}\left(\frac{l_{x}(t, h)}{2}, t\right) d \omega \geq c^{\prime}\left(\frac{l_{x}}{2}\right)^{H}$

where

$\frac{1}{A\left(\Omega\left(l_{x}\right)\right)} \iint_{\omega \in \Omega\left(l_{x}\right)} l_{y}\left(\frac{l_{x}(t, h)}{2}, t\right) d \omega$

represents the mean of all highest peaks of $l_{x}$ width and is noted $l_{y}\left(l_{x}, T\right)$.

Then from the definition of the fractal dimension related to the holder exponent [22], it can be noted that $H \approx \log l_{y} / \log l_{x}$ and therefore from Eq. (18) we can write

$\Delta\left(G_{f}, T\right)=2-\log l_{y}\left(l_{x}, T\right) / \log l_{x}$

Now if $l_{y}\left(l_{x}, T\right)<<l_{x}$ then $l_{y}\left(l_{x}, T\right)=l_{x}^{2} / 8 r_{c}\left(l_{x}, T\right)$ and we obtain the final result

$\Delta\left(G_{f}, T\right)=2-\left(\log r_{c}\left(l_{x}, T\right) / \log l_{x}\right)$.

Remarks:. Experimentally, the fractal dimension $\Delta\left(G_{f}\right)$ is obtained as a slope, by fitting in a log-log plot the discretized data $\left(\log l_{x}, \log r_{c}\left(l_{x}\right)\right)$ performed by our algorithm. If the regression line fit well, the experimental data then allow writing:

$r_{c}\left(l_{x}\right)=\alpha l_{x}^{\Delta}$

So, the relation between $l_{x}$ and $l_{y}$ becomes:

$l_{y}\left(l_{x}\right)=\beta l_{x}^{2-\Delta}, \quad \beta=1 / 8 \alpha$

An interesting properties linked to the fractal concept is the box counting method. More precisely, the box counting is a method of gathering data for analyzing complex patterns by breaking a dataset, object, image, etc. into smaller and smaller pieces, typically "box"-shaped, and analyzing the pieces at each smaller scale. When box counting is done to determine a fractal dimension known as the box counting dimension, the information recorded is usually either yes or no as to whether or not the box contained the curve or not. In our cases, we will apply similar method counting the circles. We will count the number of cases 
$N\left(r_{c}\left(l_{x}\right)\right)$ when the radius of $r_{c}\left(l_{x}\right)$ is met for a given $l_{x}$. This expression allows us to quantify the density of peaks of the surface that is fundamental in tribology (contact mechanic, wear, ...). However, the density of peaks depends also on the scale. Intuitively, for a fixed macroscopic area, the number of peaks will decrease when their radius will increase. It becomes then obvious to find the scaling law of this decrease. On the other hand, introducing the number $N\left(r_{c}\left(l_{x}, T\right)\right)$ of cases where a radius $r_{c}\left(l_{x}, T\right)$ on the profile length is met through the above algorithm (of this is the same number of count that $l_{y}\left(l_{x}, T\right)$ i.e. $\left.N\left(r_{c}\left(l_{x}, T\right)\right)=N\left(I_{y}\left(l_{x}, T\right)\right)\right)$. We have found the following results:

$\Delta\left(G_{f}, T\right)=\limsup _{l_{x} \rightarrow 0}\left(-\frac{\log N\left(l_{y}\left(l_{x}, T\right)\right)}{\log l_{x}}\right)$

and therefore

$\Delta\left(G_{f}, T\right)=\limsup _{l_{x} \rightarrow 0}\left(-\frac{\log N\left(r_{c}\left(l_{x}, T\right)\right)}{\log l_{x}}\right)$

The same reasoning as for Eqs. (22) and (23) applied to Eqs. (24) and (25), allows us to obtain the following power laws:

$N\left(r_{c}\left(l_{x}\right)\right)=\alpha^{\prime} l_{x}^{-\Delta}$

$N\left(l_{y}\left(l_{x}\right)\right)=\alpha^{\prime} \quad l_{x}^{-\Delta}$

\subsubsection{Properties}

(1) The fractal dimension is unchanged if $f$ is multiplied by a given factor.

(2) The fractal dimension is unchanged, through an $\gamma$ homothetic transform (i.e. $z^{\prime}=f(\gamma t)$ ) of the parameter $t$.

(3) If $m$ and $m^{\prime}$ denote respectively the numbers of $z$ and $z^{\prime}$ intercepts, with $m^{\prime}=\mathrm{km}$, then $N\left(r_{c}\left(z^{\prime}, x\right)\right)=k \alpha^{\prime} l_{x}^{-\Delta}$.

(4) If we do not impose the conditions $z_{i-q}<\cdots<z_{i-2}<z_{i-1}<z_{i}$ and $z_{i}>z_{i+1}>z_{i+2}>\cdots>z_{i+p}$, the Nowicki's method is then a particular case of our method, by taking different values $l_{y}=0.1 R_{m}$

\subsection{Analysis of discretisation error by simulation of Brownian profile}

A Brownian profile with fractal dimension 1.5 is generated by an algorithmic process (Fig. 3) and discretized in $10^{7}$ points. An advantage of this type of profile is to avoid the arithmetic error due to the floating point and inherent to fractal functions as Knopp or Weierstrass.

The sampling rate is equal to 1 meaning that $x_{i+1}-x_{i}=1$ and the profile is normalised to an amplitude equal to 1 on the whole interval length $L$. As it was proved, these operations do not affect the different values of the signal fractal dimension. When the algorithm is applied on curves with a number of intercepted lines $m=100$ (Fig. 1), one obtains a total of 94,026 couples of values $\left(l_{x}, l_{y}\left(l_{x}\right), r_{c}\left(l_{x}\right)\right)$ where $l_{x} \in[1 \cdots 8745294]$. As a result of the computation, the variation ranges for $l_{y}\left(l_{x}\right)$ and $r_{c}\left(l_{x}\right)$ are, respectively, [3.83 $\left.10^{-9} \cdots 0.99\right]$ and $\left[467 \cdots 1.110^{13}\right]$. As $l_{x}$ values integers on account of the discretization and according to Eq. (10), one gets a set of values $r_{c}\left(l_{x}\right)$ and $l_{y}\left(l_{x}\right)$ for a given $l_{x}$. However, if we plot the graph of the set $\left(l_{x}, l_{y}\left(l_{x}\right), r_{c}\left(l_{x}\right)\right)$ and determine the fractal dimension, the slope of the regression line will then be calculated for a great part of the small $l_{x}$ values. If we make the regression from the set of data, one can obtain $N\left(l_{y}\left(l_{x}\right)\right)=N\left(r_{c}\left(l_{x}\right)\right)=30902 l_{x}^{-1.42}$ (Fig. 4) with a good coefficient of correlation $r_{\text {corrrel }}=0.98$.

Consequently, we decide to calculate the value of the mean $\left(\bar{l}_{y}\left(l_{x}\right), \bar{r}_{c}\left(l_{x}\right)\right)$, for each $l_{x}$. Fig. 5a represents the variation of $\log \bar{r}_{c}\left(l_{x}\right)$ versus $\log l_{x}$ and the corresponding linear equation is given by:

$\log r_{c}\left(l_{x}\right)=2.756_{ \pm 0.01}+1.488_{ \pm 0.004} \log l_{x}$

with regression coefficient $r=0.998$. The studied test proves that this slope is not statistically different from the theoretical fractal dimension equals to 1.5 for the Brownian motion. Four remarks could then be stated from the analysis of the residual regression:

(i) $r_{c}\left(l_{x}\right)$ is overestimated for small $l_{x}$ values and increases exponentially with $l_{x}$ : there are estimation errors on $r_{c}\left(l_{x}\right)$.

(ii) The $l_{x}$ are approximately Gaussian distributed values with a high right tail.

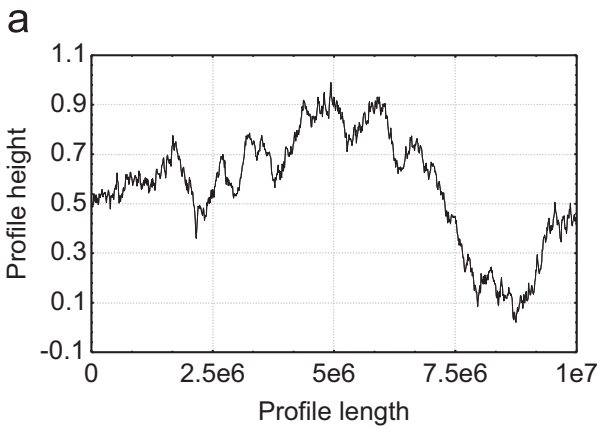

b

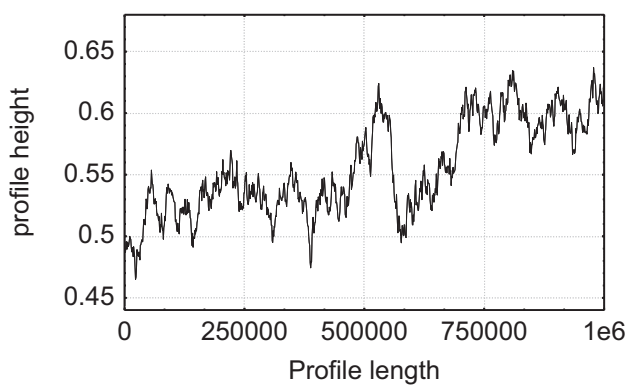

C

d
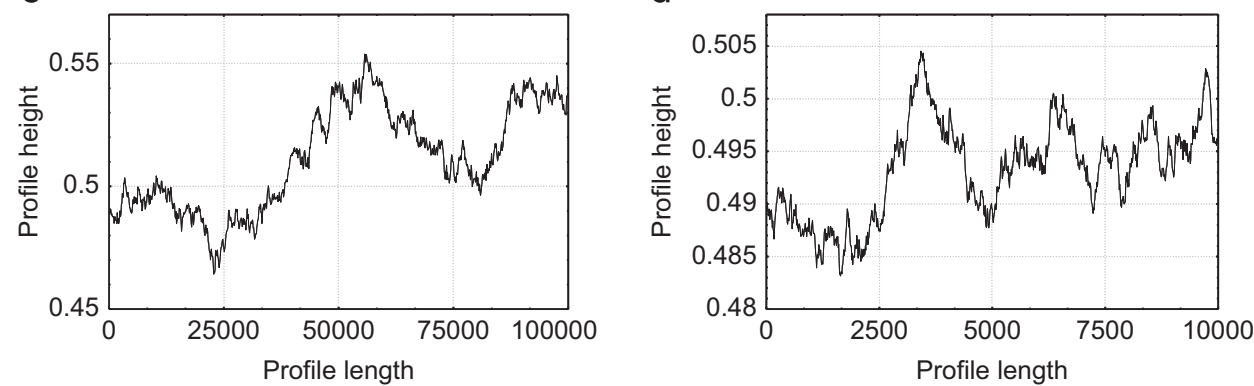

Fig. 3. Brownian profile generated using $10^{7}$ discretisation points (a) and different zooms at the origin: $\times 10$ (b), $\times 100(\mathrm{c}), \times 1000$ (d) 


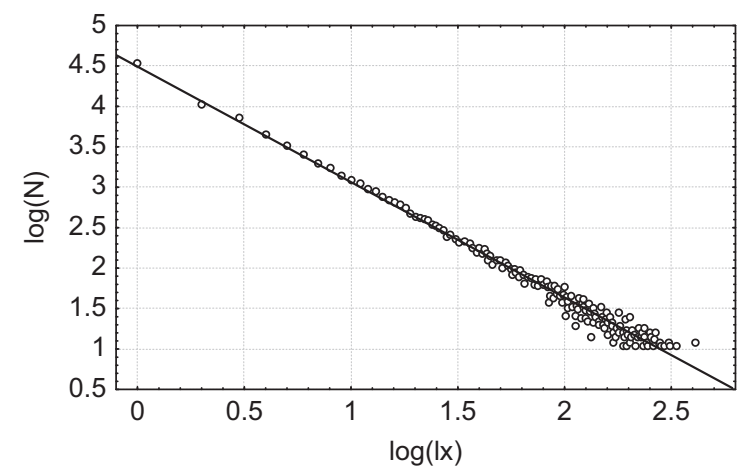

Fig. 4. Evolution of the number of curvature radii $\log N\left(r\left(l_{x}\right)\right)$ of the profile shown in Fig. 3a, estimated at a $l_{x}$ values versus the $\log l_{x}$ value.
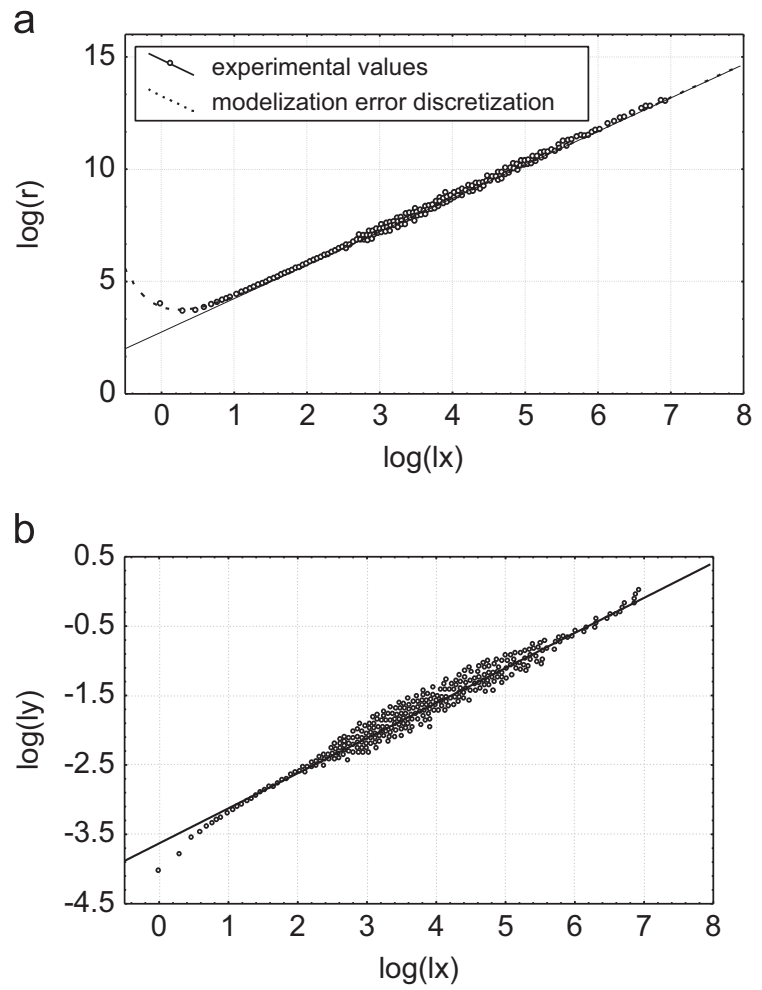

Fig. 5. Evolution of the curvature radius $\log r\left(l_{x}\right)$ of the profile shown in Fig. 3a, estimated at a $l_{x}$ values versus the $\log l_{x}$ value (a). The thin line corresponds to the equation $\log r_{c}\left(l_{x}\right)=2.756_{+0.01}+1.488_{+0.004} \log l_{x}$ and the dashed one to the corrected method with $\log r_{c}\left(l_{x}\right)=2.732_{ \pm 0.01}+1.494_{ \pm 0.003} \log l_{x}+1.14_{ \pm 0.1} /\left(n_{x}-1\right)$ (where $n_{x}$ is the number of $d_{x}$ intervals used to calculate $l_{x}$ ). The graph (b) represents the evolution of the height curvature radii $\log l_{y}\left(l_{x}\right)$ versus the $\log l_{x}$ value.

(iii) The $r_{c}\left(l_{x}\right)$ dispersion increases with $l_{x}$.

(iv) The linear approximation is accurate for the values of $l_{x}$ that are not too small.

(v) Appendix B presents other validations with function possessing different fractal dimensions.

\section{Part 2-Model application to physical processes}

\subsection{Example 1-Analysis of peaks radius flattening} during boundary lubricated friction

\subsubsection{Metal forming experimental device}

Experimental analysis of aluminium strip reduction by drawing process has been carried out using device developed by Bech
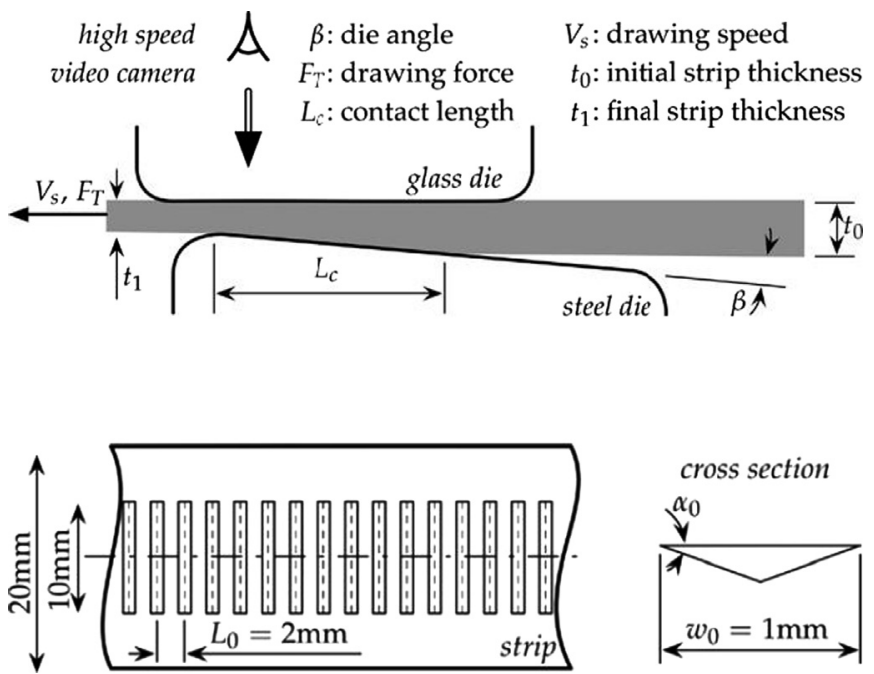

Fig. 6. Schematic diagram of experimental device used for drawing process after [31].

et al. [23] at Technical University of Denmark. It consist of two working tools, i.e. glass and steel dies rigidly mounted in experimental setup assuring $20 \%$ reduction in thickness of aluminium strip. Steel die is inclined of $\beta=3^{\circ}$ and its position is adjusted so that deformation zone can be observed through die made of transparent hardened glass. Glass die is in form of circular disc with $50 \mathrm{~mm}$ diameter and $11 \mathrm{~mm}$ thick. The drawing speed $V_{s}$ can be adjusted and in this study speed of $5 \mathrm{~mm} / \mathrm{s}$ has been used. Schematic diagram of experimental device is presented on Fig. 6. The material used for strip is semi-hard aluminium AISI 1050 H24. The strips have been cut from a metal sheet of $2 \mathrm{~mm}$ into samples of the following dimensions: $450 \times 20 \times 2 \mathrm{~mm}$. One side of the strip have been textured. Mesoscopic pockets were manufactured using Electro Discharge Machining (EDM) technique. Pockets in shape of about $10 \times 2 \mathrm{~mm}$ grooves were manufactured, initial angle of side walls creating the pockets were about $\alpha_{o}=10^{\circ}$. Initial roughness inside the pockets were significantly higher than roughness on flat surface between the pockets (Fig. 7). Viscosity of used mineral oil was 0.5954 Pa s.

\subsubsection{Surface roughness measurement and analysis}

Surface roughness were measured using Zygo NewView 7300 interferometric profiler. Vertical Scanning Interferometry (VSI) was used to measure the surfaces. The measurement principle is that unfiltered white light beam is split in two. Half of a beam is directed through a microscope objective and reflected from the surface and half is reflected from the reference mirror. When reflected beams combine together they produce interference fringes, where the best-contrast fringe occurs at best focus. In VSI mode the objective moves vertically to scan the surface at various heights. A 3D surface is reconstructed by analysis of fringes at every pixel. VSI mode uses algorithm to process fringe modulation data from the intensity signal to calculate surface heights. Obtained resolution will therefore depend on a precision of $z$ axis positioning. In case of Zygo NewView 7300 instrument a piezoelectric stage with range of $160 \mu \mathrm{m}$ is used to refine the height resolution going down to less than a $1 \mathrm{~nm}$. Spatial resolution will depend on camera size and used objective. In our case a several scans were made and stitched together to cover an area of $2 \times 8 \mathrm{~mm}$ with spatial resolution $(X, Y)$ resolution of $1.1 \mu \mathrm{m}$.

On the surface measured after the lubricated drawing test has been performed, different zones on tribological contact can be defined. As shown on Fig. 7 on both sides of deformed plateau two 


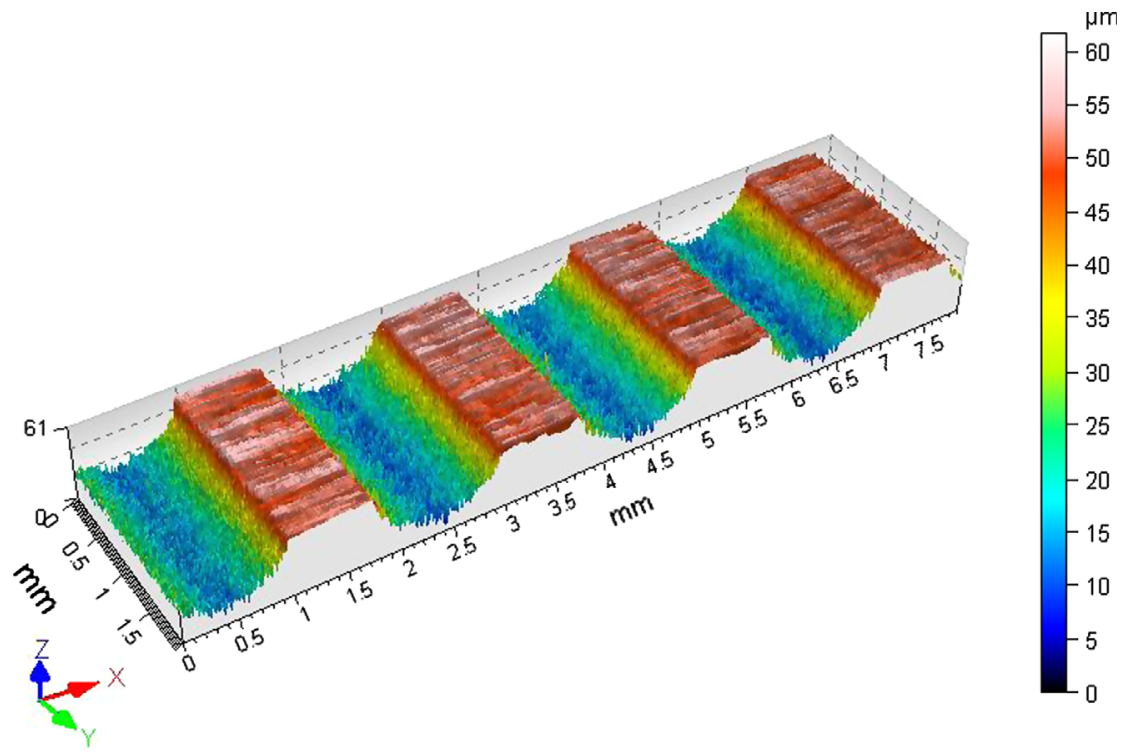

$$
\begin{aligned}
& 1 \text { - Pocket valley } \\
& 2 \text { - Plateau inlet } \\
& 3 \text { - Plateau } \\
& 4 \text { - Plateau tail } \\
& \text { 1' - Pocket valley }
\end{aligned}
$$

$\mu m$

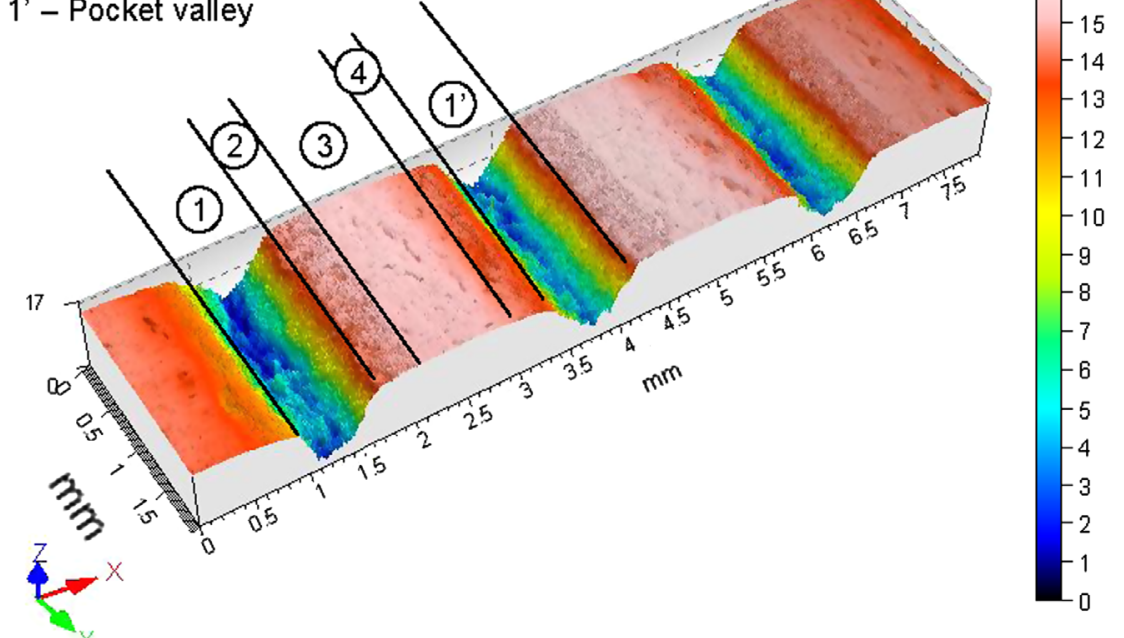

Fig. 7. Initial textured surface with pockets prepared by electro erosion process and final morphology after drawing test, lubricated by mineral oil, different zones created on textured surface can be defined.

new zones can be found: zones 2 and 4. Initially those surfaces were inside the cavities pockets and due to elastoplastic deformation they form now part of a plateau. However, due to contact lubrication there was only partial contact between plateau and glass tool. In such contact, surface roughness will change leaving a specific signature of tribological process. Analysis of surface roughness can reveal that signature. However, direct analysis of 3D surface morphology is computationally more expensive and less robust than 2D analysis. Therefore to analyse surface roughness specific technique of transition from 3D surface to 2D surface profiles analysis has been developed. It consist of generation a series of 2D profiles in vertical direction (Fig. 8) from 3D surface. Once the profile are extracted different parameters can be calculated from individual profiles. Evolution of that parameters can be plotted in function of initial position of extracted profiles. Hence, every point on a graph corresponds to roughness parameter calculated from one vertical profile (Fig. 8).
Analysis of several different roughness parameters, revealed that only two parameters are able to clearly distinguish created zones on tested surface. The first one is developed length of the roughness profile $R_{L o}$ (Fig. 8) and the second is our new parameter of profile curvature radius $r_{c}$ (Fig. 9 and Fig. 10). In this example the $r_{c}$ is calculated as an average value of $r_{c}$ and will be named $\bar{r}_{c}$ for randomly chosen $l_{x}$ lines. This approach will produce single parameter $r_{c}$ which will be scale independent. One characteristic value of $r_{c}$ start to dominate, therefore sufficient sampling number should be chosen to obtain more accurate estimation of dominant curvature radius for a given surface profile. Evaluating the value of $\bar{r}_{c}$ before and after tribological process create characteristic signature of surface.

For comparison also an evolution of arithmetic mean value of roughness profile $R_{a}$ has been plotted however zones 2 and 4 cannot be distinguished using this simple amplitude parameter. $R_{L o}$ is able to show zones 2 and 4 however contrary to $r_{c}$ the value 

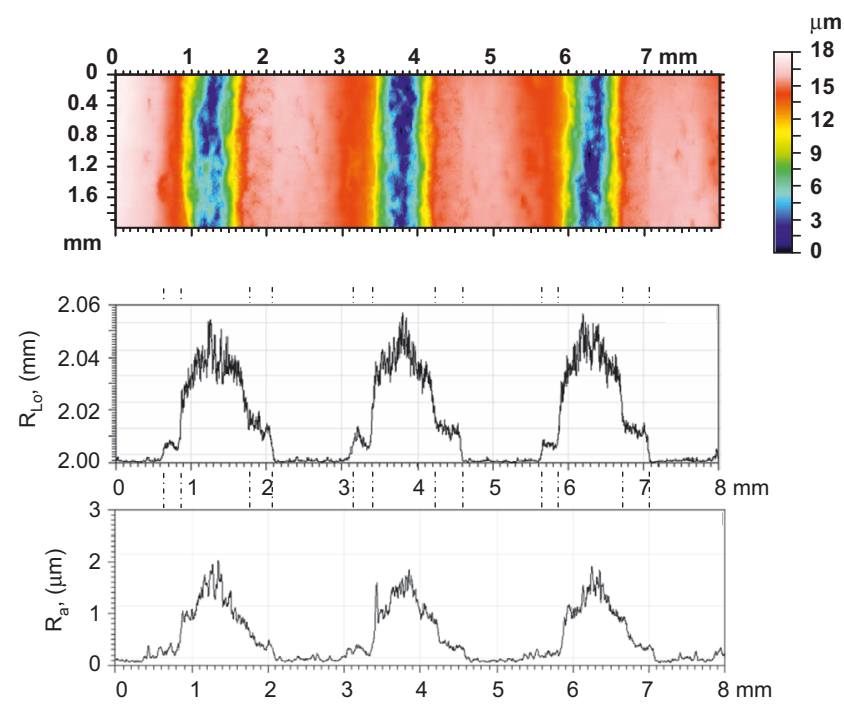

Fig. 8. Analysis of roughness parameters $\left(R_{a}\right.$ and $\left.R_{L o}\right)$ calculated on individual 2D profiles extracted from surface in vertical direction, every point on bottom graphs corresponds to roughness parameter calculated from one vertical profile from surface on top.

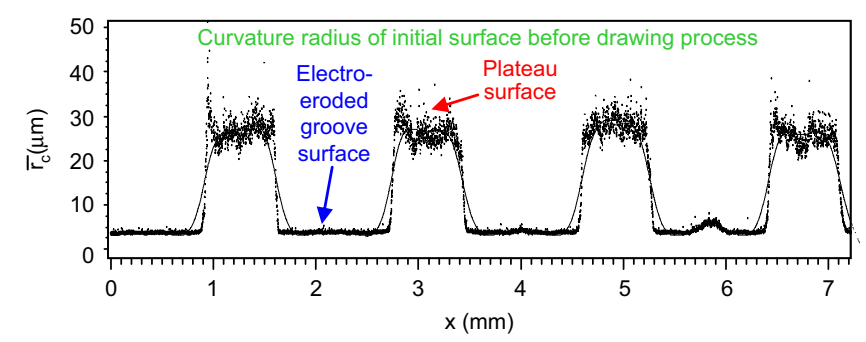

Fig. 9. Averaged curvature of peaks roughness $\bar{r}_{c}$ calculated on initial profile before drawing process, showing very regular sharp peaks in elecroeroded zone ( $\operatorname{small} \bar{r}_{c}$ around $6 \mu \mathrm{m})$ and higher peaks value on plateau $\left(\bar{r}_{c}\right.$ around $\left.30 \mu \mathrm{m}\right)$.

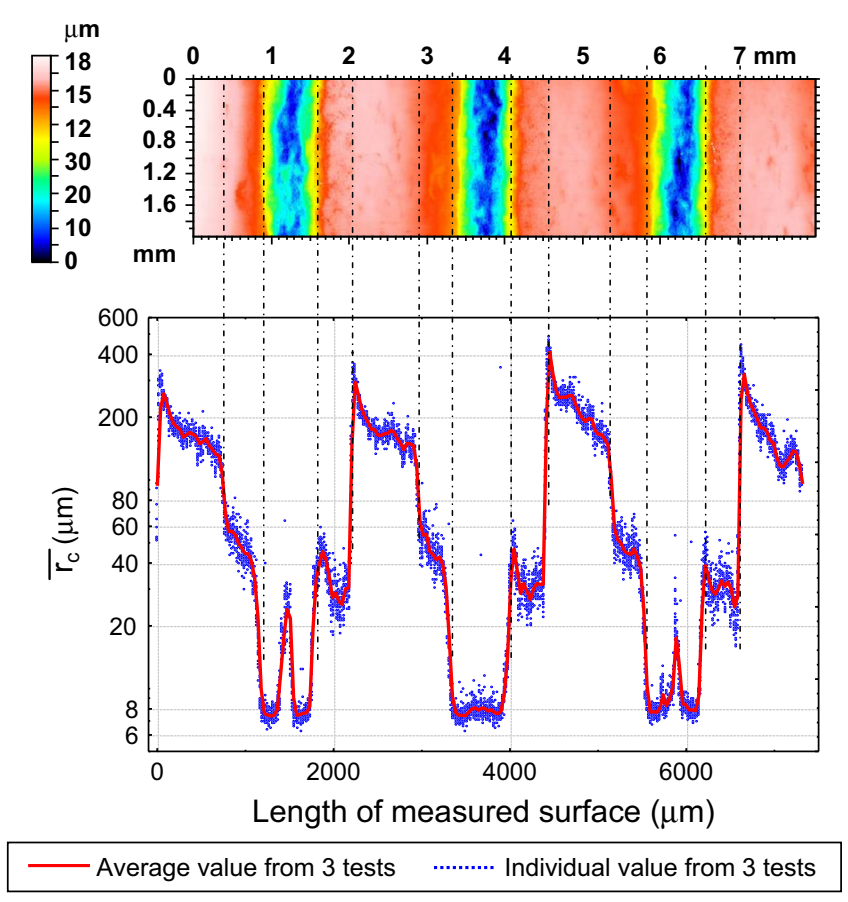

Fig. 10. Analysis of average roughness peaks curvature radius $\left(\bar{r}_{c}\right)$ calculated on individual 2D profiles extracted from surface in vertical direction, every point on bottom graphs corresponds to roughness parameter calculated from one vertical profile. at the plateau do not present any variation. In Fig. 9 an initial value of $r_{c}$ parameter calculated on initial surface is analysed. Presented in Fig. 10 evolution of parameter $r_{c}$ shows very well different zones and also variations of the parameter across the zone. High value of curvature radius of roughness profile at the left hand side of the plateau (zone 3) confirm more severe contact conditions in this area and flattening of the peaks.

Variation of average roughness curvature before (Fig. 9) and after (Fig. 10) drawing process can be considered as characteristic signature of tribological contact.

\subsubsection{Multiscale decomposition}

In this section the multiscale decomposition of the radius curvature analysis is performed. One of the major interest in our method is to presents a multiscale evaluation of the radii curvature. As claimed in this paper, the curvature radius depends on the scale. It could then be obvious to represents the effect of drawing conditions as the multiscale variation of the radius curvature.

According to Eq. (22) and using a new notation involved by our study, radius curvature is equals to

$$
r_{c}\left(l_{x}, p\right)=r_{c 0}(p)\left(l_{x}(p) / l_{x}^{0}\right)^{\Delta(p)}
$$

where $p$ represents the position on the measured surface along the drawing direction, $l_{x}^{0}$ the sampling length unit. In this case $r_{c 0}(p)$ can be seen as an unscaled curvature radius i.e. independent of the length $l_{x}(p)$. Fig. 11 presents the evolution of $r_{c 0}(p)$ and Fig. 12 shows evolution of $\Delta(p)$. Therefore, presented in Fig. 13 multiscale variogram shows multiscale decomposition like those met in the wavelet decomposition allowing visualizing all multiscale features of the radius curvature. More detailed analysis of those aspects will be published in a follow-up paper where different drawing conditions will be analysed.

Different part of surface depending on local contact conditions will have different value of $r_{c}$, fractal dimensions and unscaled radius curvatures. In case when the contact conditions are unknown, this parameter can be used to determine the regime of lubrication or zones of direct contact between contacting surfaces. Therefore, it can be a very robust tool for contact rheology by roughness analysis in tribological applications.

\subsection{Example 2-Analysis of stylus tip artifacts introduced during surface measurement}

Surface data of a fractal nature may be gathered in a very wide variety of ways. All involve a certain amount of smoothing or degradation of the true surface data according to the recording method. In laser profilometry, devices provide a smoothing measuring the surface height via the interference patterns of the reflected height from a narrow beam. With mechanical profilers, the stylus tip curvature radius makes a smoothing effect on the surface and the information narrower than the stylus tip cannot be recorded. Since the smoothing effect is highly non-linear, it becomes very hard to estimate it on the original data. The basic idea we propose in this paper is to apply the theory of curvature radius to detect the scale on which the measurement system introduces a smoothing artefact on the data measurement. In fact, the smoothing effect will increase the curvature radius on the scale measurement.

\subsubsection{Analysis of an experimental measurement}

In a great number of roughness study using tactile profilometry, it was shown that the curvature radius $r_{c}$ calculated from the Nowicki's formulation was not a discriminate parameter for physical phenomena. We also have noticed that the values of $r_{c}$ will depend on the sampling length. As an application, a pure 


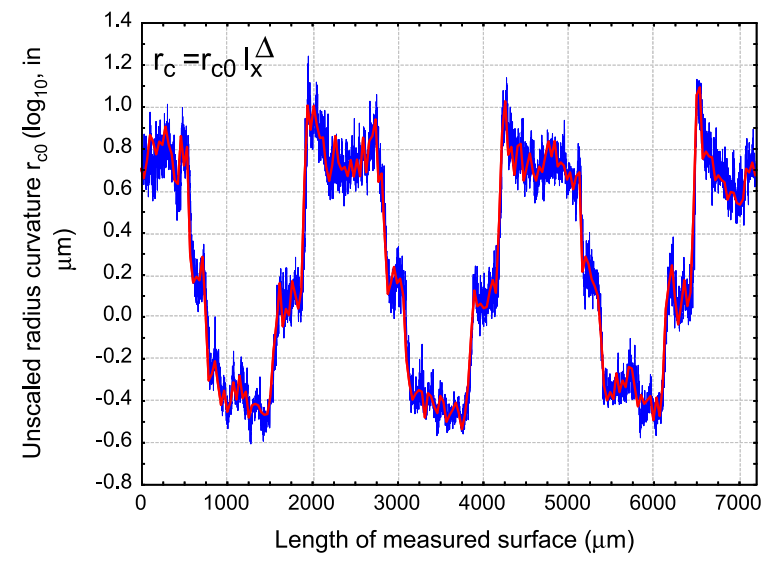

Fig. 11. Evaluation' of the unscaled curvature radius $r_{c 0}(p)$ versus the measured surface along the drawing direction $p$.

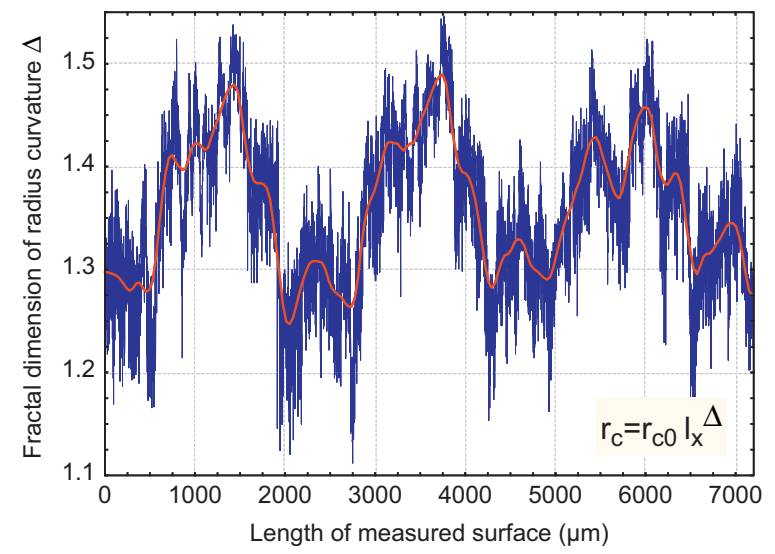

Fig. 12. Evaluation of the fractal dimension $\Delta(p)$ versus the measured surface along the drawing direction $p$.

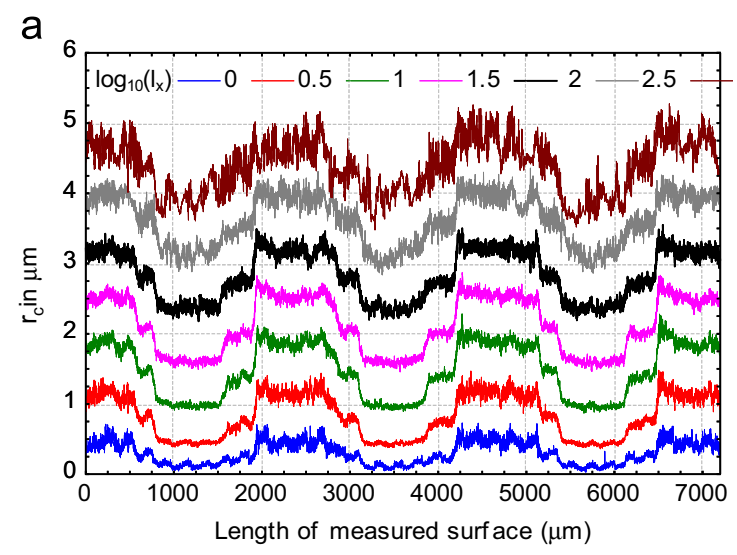

b

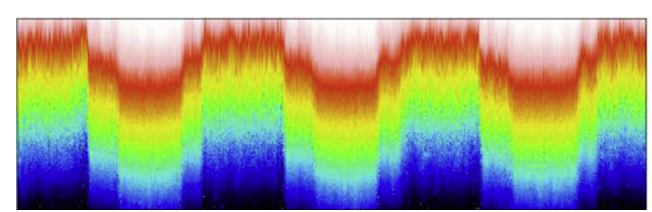

Fig. 13. Multiscale decomposition of $r_{c}\left(l_{x}, p\right)$, (a) decomposition graph, (b) decomposition map.

Aluminium sheet is polished with different paper grades: 80,120 , 220 and 500 . Then 30 bi-dimensional profiles are recorded using a tactile profiler with a $10 \mu \mathrm{m}$ stylus radius.
Fig. 14 shows the variation of $\log r_{c}\left(l_{x}\right)$ versus $\log l_{x}$ and Fig. 15a shows an example of a grade 500 polished surface. As it could be shown on this last one, a cross over appears around $r_{c}\left(l_{x}\right)=10 \mu \mathrm{m}$. Below this critical value, $r_{c}\left(l_{x}\right)$ seems to be constant and over it, $r_{c}\left(l_{x}\right)$ follows the power law given by Eq. 22. As it could be observed, the curvature radius is lower than for a smaller grain

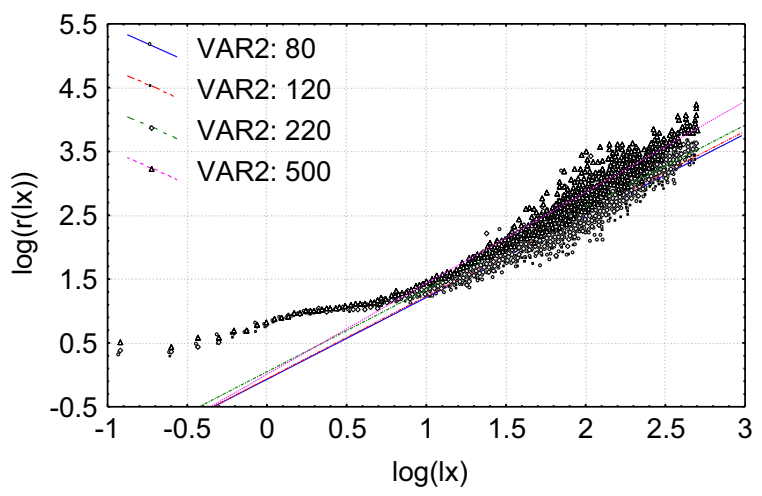

Fig. 14. Evolution of the curvature radius $\log r\left(l_{x}\right)$ versus $\log l_{x}$ for pure Aluminium sheet surface polished with different paper grades 80,120, 220 and $500\left(l_{x}\right.$ and $r\left(l_{x}\right)$ in $\mu \mathrm{m})$.
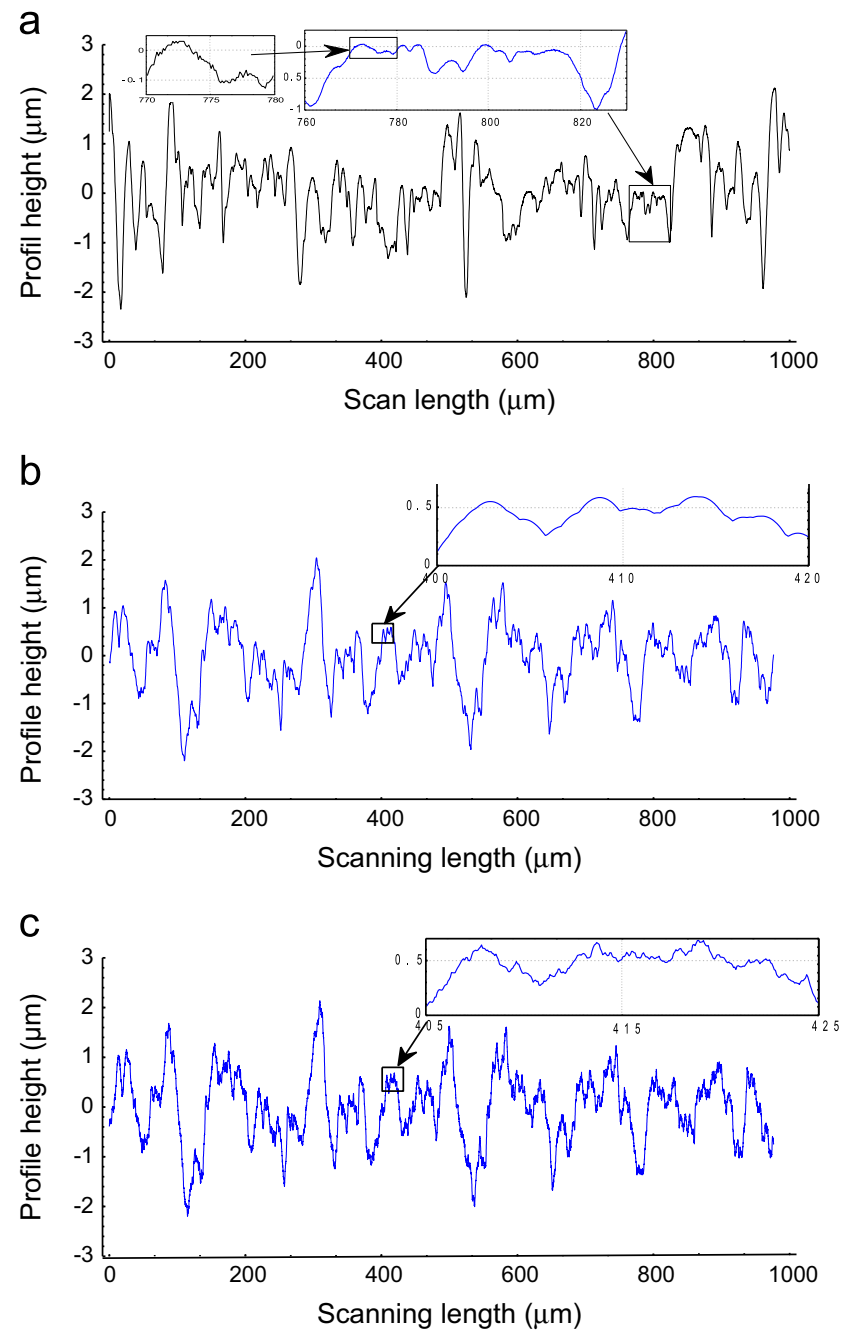

Fig. 15. (a) Profile of a pure Aluminium sheet surface polished with paper grade 500 with a $10 \mu \mathrm{m}$ stylus tip curvature. (b) Simulation of the scanning of the profile $\mathrm{c}$ with a $10 \mu \mathrm{m}$ stylus tip curvature. (c) Simulation of profile a by stochastic circle function. 
size. For different paper grades, the cross over related to $r_{c}\left(l_{x}\right)$ is the same but the abscissa $l_{x}$ increases with the grain size. This fundamental relation shows that whatever the roughness amplitude of polished surfaces ( $R_{a}$ varying from 1.66 to $0.02 \mu \mathrm{m}$ ), the stylus size effect will give a same constant value of the curvature radius. The stylus size effect is greatly discussed in the bibliography [24-28], however no invariance parameter was found in the roughness measurement until now.

\subsubsection{Fractal simulation of experimental measurements}

We have decided to write an algorithm that simulates the stylus effect that we would apply to the Weierstrass functions defined only by an amplitude factor $A$ and the Hölder exponent $H$ (Eq. (19)). This algorithm simulates physically the scanning effect without using mathematical considerations on the profile and it is then well adapted for fractal curves. By an inverse method, the scaling factor and the fractal dimension are adjusted to experimental data to reproduce profiles that look like the polishing profiles after the stylus scanning effect was simulated with a curvature radius of $10 \mu \mathrm{m}$. Fig. 15b shows the simulated profile corresponding to the experimental one (Fig. 15a) including the stylus integration simulation algorithm on the original simulated (Fig. 15c). Table 1 presents the roughness parameters calculated on experimental profiles, as well as the simulated profiles and the stylus integration simulation algorithm profiles $\left(R_{a}\right.$ : mean roughness amplitude, $R_{t}$ : Range amplitude, Peaks: number of peaks by inch, $D$ : fractal dimension calculated by the ANAM method $[29,30]$.

From this analysis the following remarks can be stated:

(i) Although our inverse method only use two parameters $A$ and $H$, the experimental roughness parameters, the simulated ones and others are statistically equal. This point implies the following particular observations:

(a) Our original fractal model is adequate for some complex worn surfaces such the polished ones with only two parameters. The mechanism seems then to be described by an amplitude phenomenon, a "circle base", stochastic components and finally the fractal dimension.

(b) The fractal dimension estimation calculated by the ANAM method seems to be very pertinent because when the estimated fractal dimension and the theoretical one are equal, the frequency roughness parameters are then equal.

(ii) The Fractal dimension of simulated surfaces with stylus integration gives less information than the original one; stylus integration leads to see the surface more Euclidean than the reality, as a consequence of the smoothing effect. The underestimation of surface fractal dimensions measured with a stylus is also confirmed by the decrease of the number of peaks characterizing a "less" fractal surface.

(iii) For a stylus radius not too-large (less than $10 \mu \mathrm{m}$ ), the amplitude parameters are quite constant. The stylus effect does not fundamentally destroy them. We then plot in Fig. 16. $\log r_{c}\left(l_{x}\right)$ versus $\log l_{x}$ for the three categories of profiles (means

Table 1

Comparison of roughness parameters calculated on measured and modelled profiles $\left(R_{a}\right.$ : mean roughness amplitude, $R_{t}$ : total range amplitude, Peaks: number of peaks by inch, $D$ : fractal dimension).

\begin{tabular}{lccc}
\hline $\begin{array}{l}\text { Roughness } \\
\text { parameters }\end{array}$ & $\begin{array}{l}\text { Measured } \\
r=10 \mu \mathrm{m}\end{array}$ & $\begin{array}{l}\text { Modelled } \\
r=10 \mu \mathrm{m}\end{array}$ & $\begin{array}{l}\text { Modelled } \\
r=0 \mu \mathrm{m}\end{array}$ \\
\hline$R_{a}(\mu \mathrm{m})$ & 0.63 & 0.64 & 0.66 \\
$R_{t}(\mu \mathrm{m})$ & 4.64 & 4.64 & 4.32 \\
Peaks/inch & 820 & 814 & 1186 \\
$D$ : Fractal dimension & 1.08 & 1.09 & 1.23 \\
\hline
\end{tabular}

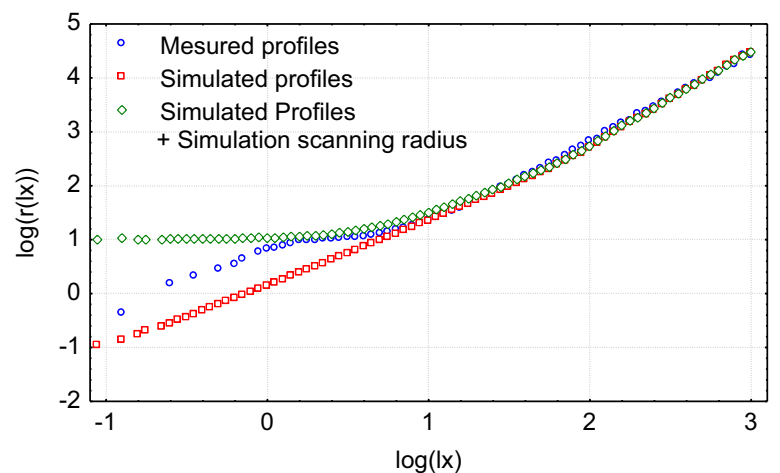

Fig. 16. Evolution of the curvature radius $\log r\left(l_{x}\right)$ versus $\log l_{x}$ for profile shown in Fig. 15a, Fig. 15b and Fig. 15c $\left(l_{x}\right.$ and $r\left(l_{x}\right)$ in $\left.\mu \mathrm{m}\right)$.

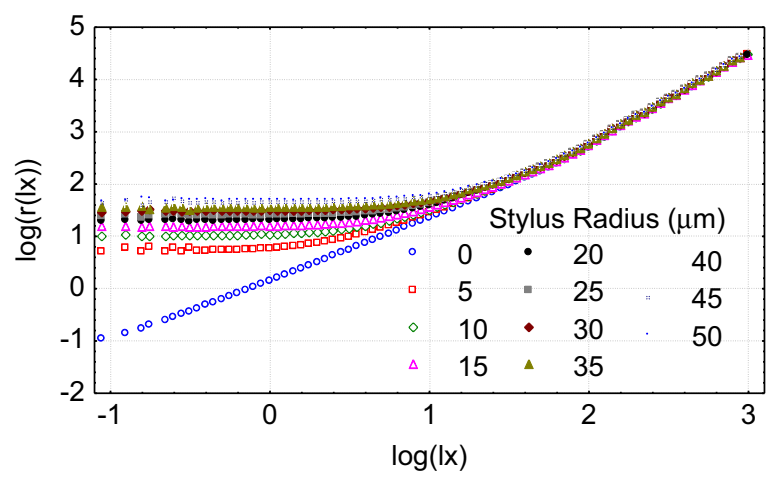

Fig. 17. Evolution of the curvature radius $\log r\left(l_{x}\right)$ versus $\log l_{x}$ for profile shown in Fig. 15c with different scanning simulation of the profiler stylus tip curvature varying in the range 0 to $100 \mu \mathrm{m}\left(l_{x}\right.$ and $r\left(l_{x}\right)$ in $\left.\mu \mathrm{m}\right)$.

values of 30 profiles). The following remarks will then be stated:

(a) For $l_{x}>30 \mu \mathrm{m}$, all points are confounded in a linear log-log relation meaning that:

(1) There is no quantified stylus effect,

(2) Models and simulated surfaces are similar in a great range of scale,

(3) Hölderian and anti-Hölderian hypotheses on experimental profiles are respected.

(b) Both simulated stylus and experimental profiles present a step at the value $10 \mu \mathrm{m}$ that is exactly the curvature radius: our method allows us to detect the stylus effect and furthermore, allows us to quantify the curvature radius of the profiler. As consequence, this method then allows us to give the critical threshold concerning $l_{x}$ and the value $l_{y}$ under which the measurement effect could affect a metric value constructed on the signal. This aspect is a very important feature in the topographic measure area.

(c) For the measured surface, if $l_{x}<2 \mu \mathrm{m}$ the curvature radius increases linearly in the $\log -\log$ plot with $l_{x}$. By analysing more precisely experimental data, it could be observed that a white noise due to the numeric analogical conversion with low amplitude is present in the profile. As we have shown in what precedes, this fact explains this linear tendency. The method proposed in this paper then allows us to detect high frequencies components and also to quantify the amplitude range for which the measure could be influenced. We will now verify for different stylus radii the relation between the discretization steps related to the curvature radii of the profile and the stylus radius. All 100 profiles on which stylus integration algorithms are applied are then simulated. Curvature radii are so estimated by our method and Fig. 17 shows this variation. 


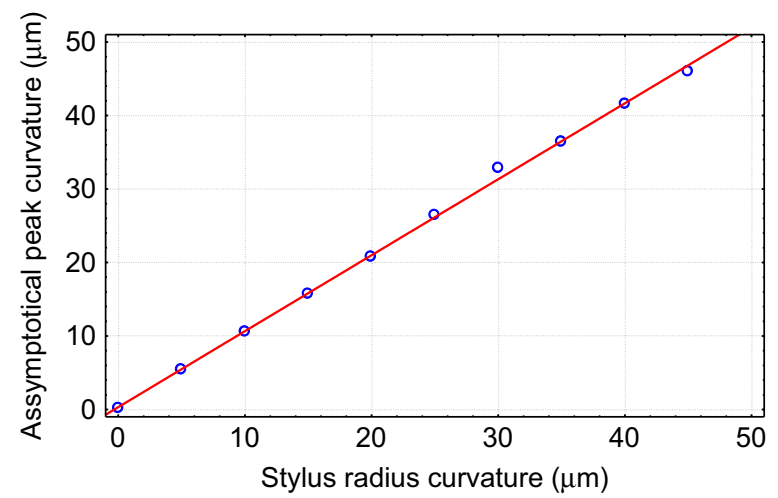

Fig. 18. Plot of the asymptotic peak curvatures of Fig. 17 versus the profiler stylus tip curvature.

We then detect the step by taking only the radii values respecting the relationship $\log r_{c}\left(l_{x}\right)>1+\log l_{x}$ with $\log l_{x}<1.5$.

On the other hand means values $r_{c}\left(l_{x}\right)$, called $r_{c}^{T}(\rho)$ are calculated for all values of $l_{x}$ for each stylus radius $\rho$ and $r_{c}^{T}(\rho)$ is so plotted versus $\rho$ (Fig. 18). By linear regression analyses, one then obtains:

$r_{c}^{T}(\rho)=0.5_{ \pm 1}+1.014_{ \pm 0.018} \rho$

with a correlation coefficient $r_{\text {correl }}=0.9986$.

\section{Conclusions}

A fractal approach of the curvature radius, on the basis of simulations, experimental measurements and analytical developments is presented in this paper. The proposed method generalizes the Nowicki's approach and then supplies a new tool overtaking the classic limits of the curvature radius analysis. It presents, in particular, an original advantage in the taking into account the highly non-linear stylus smoothing effect during the data gathering. New method is applied to tribological contact in metal forming process. Calculations of curvature radius along the tribological surface can be used to estimate the flattening effect of peaks curvature and therefore determine the areas of direct or boundary contact in measured tribological surfaces. This method can be of particular interest in lubricated contact to analyse lubrication regime and reveal the contact history. This technique showed to be robust in topographical contact analysis. Calculated average curvature of roughness peaks $\left(r_{c}^{\prime}\right)$ can be used to estimate topographical signature of tribological contact.

\section{Acknowledgements}

Authors would like to thank Prof. Niels Bay from Technical University of Denmark for providing experimental support for tribological drawing tests and stimulating scientific discussions.

K.J. Kubiak would like to thank Prof. L. Dubar, Prof. A Dubois and other members of staff of the Laboratory TEMPO at the University of Valenciennes for inviting him for 'visiting professorship', and very fruitful collaboration.

\section{Appendix A. The Definition of a peak}

We will now consider a peak as $z_{i-q}<\cdots<. z_{i-2}<z_{i-1}<z_{i}$ and $z_{i}>z_{i+1}>z_{i+2}>\cdots>z_{i+p}$, therefore $\tilde{l}_{x}=x_{i+p}-x_{i-q}$ and $\tilde{l}_{y}=z_{i}-$ $\left(z_{i+p}+z_{i-q}\right) / 2$. It is then possible to compute a $r_{c}$ value. With this

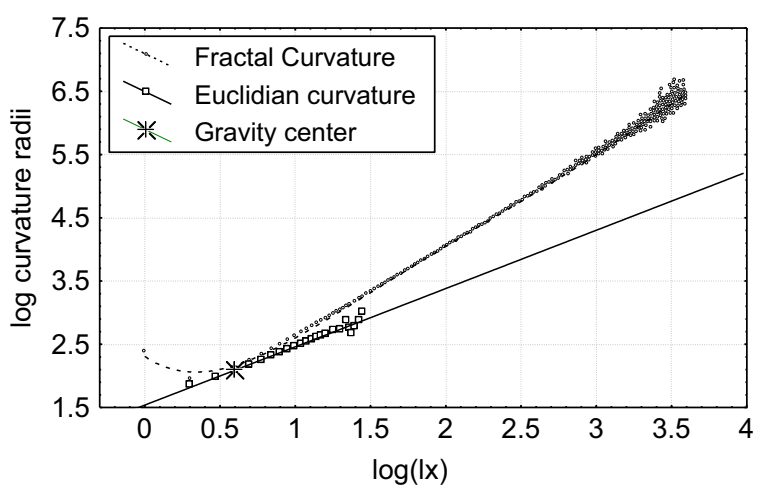

Fig. A1. Evolution of the fractal radii curvature $\log r\left(l_{x}\right)$ and Euclidian radii curvature $\log \tilde{r}\left(l_{x}\right)$ versus $\log l_{x}$ for a Brownian motion.

formulation, peak will be seen as a non fractal structure. We will name this peak as Euclidian peak $\tilde{r}_{c}$. According to Eq. (23) by letting $\Delta=1$ (non fractal structure), one would have $l_{y}\left(l_{x}\right)=\beta l_{x}$ and thus for any fractal dimension of the initial curve. This mean that giving an ideal definition of the radii curvature will pass over the fractal dimension of the curve and peaks become homothetic. We will now verify this assumption by simulate 1000 Brownian curves with a sampling length equal to unity and discretized in 4000 points. Amplitude of each curves are normalize to unity. Then we calculate the values of $\tilde{l}_{x}, \tilde{l}_{y}$ and $\tilde{r}_{c}$. We find the following relation $\tilde{l}_{y}=30_{ \pm 3} \tilde{l}_{x}^{1.01_{ \pm 0.04}}$ with $R=0.999$ proving that this structure is Euclidean. In Fig. A1, we have plotted the evolution of the mean value of curvature radii calculated from the fractal $\left(r_{c}\right)$, and non fractal approach $\left(\tilde{r}_{c}\right)$ versus the values of $l_{x}$ and $\tilde{l}_{x}$. Then regressions are proceeded on the two samples. The distribution of the number of peaks measured with the Euclidean method is plot on Fig. A1. One find the mean of $\tilde{l}_{x}$ equals to 4 and the number of $\tilde{l}_{x}$ decrease exponentially. On the fractal curve, the probability to have $99.5 \%$ of detected radii $\tilde{r}_{c}$ by this classical algorithm are under 10 sampling length. The gravity centre of $\tilde{l}_{y}$ and $\tilde{r}_{c}$ is marked by the star on the graph. This point is on the curves of the fractal radii described by Eq. (22). What is then the physical meaning of that point? In fact, this Euclidian algorithm is only a particular case of our method. It fixe a critical length and measure all the homothetic peaks. This approach validates our original method of determination of radii curvature.

\section{Appendix B. Validation of the method on simulated fractal curves}

To test the efficiency of our algorithm, we have chosen to calculate the curvature radius for curves with different fractal dimensions: a perfect white noise $(\Delta=2)$ and a Weierstrass Function with different fractal dimension.

\section{The white noise}

It seems not obvious to calculate a curvature radius for a white noise. In fact, we can find that $R$ does not exist. We start from analysis of $R$ properties: is $R$ infinite? If that is the case, could the surface be considered as a plane? Is in this case the radius curvature $\tilde{r}_{c}$ still defined? How can we henceforth apply our reasoning to these two questions? In fact, that depends on the scale of observation. Indeed, according to Eq. (23), the condition allowing the definition of the curvature radius implies $l_{x} \geq \beta$ where $\beta$ is a critical length where the peak is defined. Therefore, if $l_{x}<\beta$ 
neither $r_{c}\left(l_{x}\right)$ nor the relation $r_{c}\left(l_{x}\right) \propto l_{x}^{\Delta}$ are defined according to our formulation. On another hand, we obtain in this case $r_{c}\left(l_{x}\right) \propto l_{y}$ that seems coherent for a white noise. For that reason the curve appears as being more flat for a large scale of observations if its fractal dimension is high. It is particularly interesting to analyse

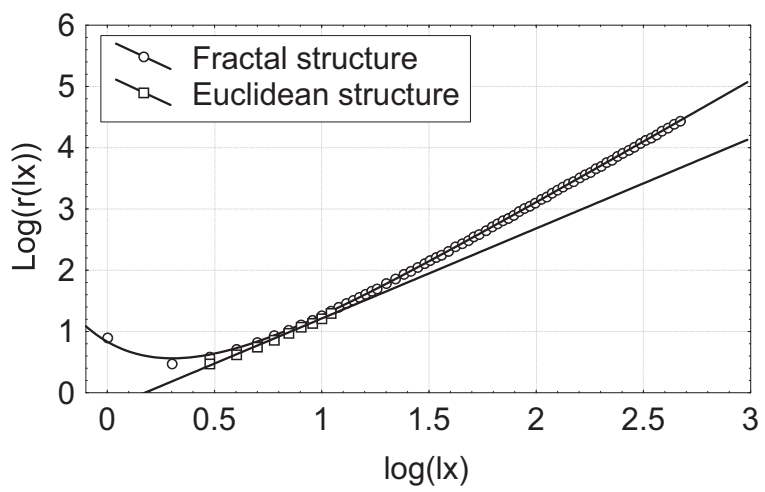

Fig. B1. Evolution of the fractal curvature radius $\log r\left(l_{x}\right)$ and Euclidean curvature radius $\log \tilde{r}\left(l_{x}\right)$ versus $\log l_{x}$ for a white noise.
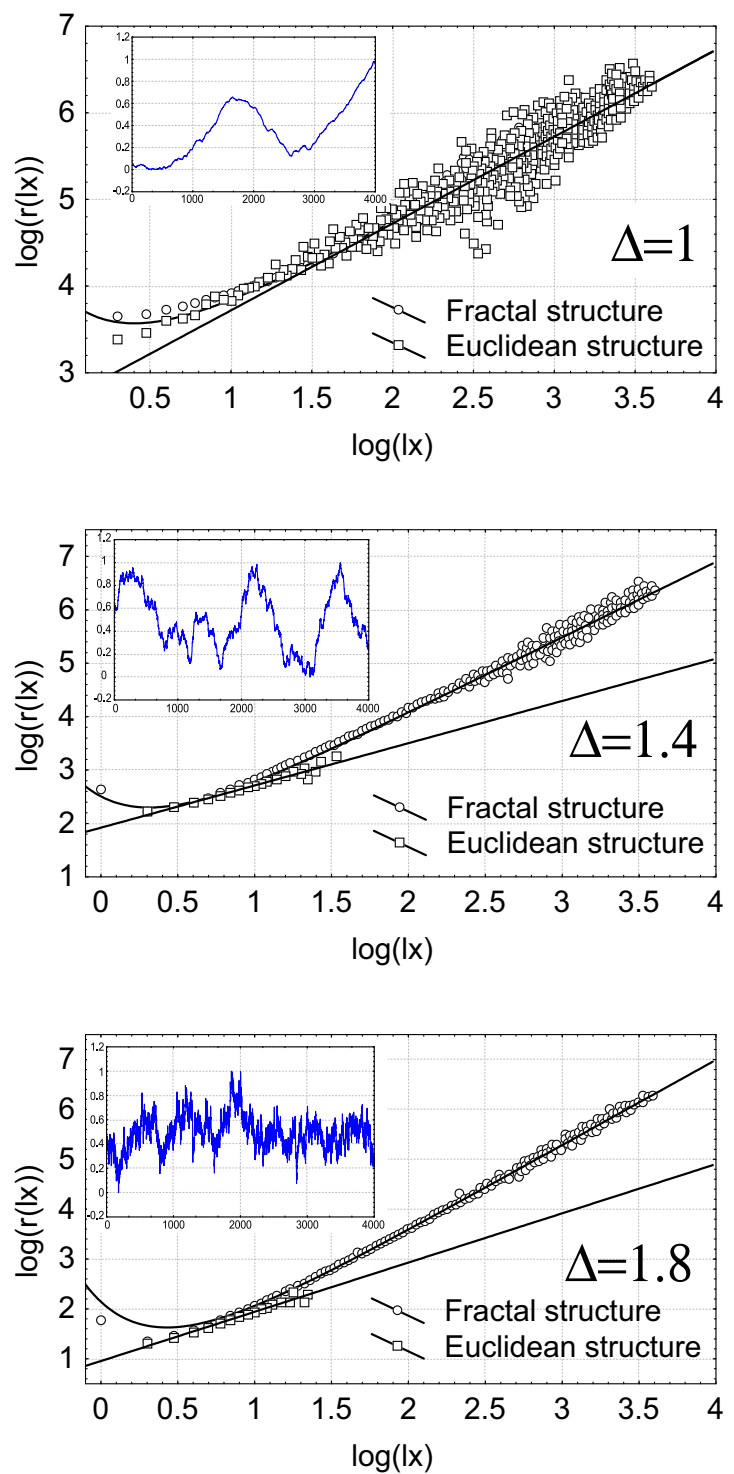

the calculus of $\tilde{r}_{c}$ or $r_{c}$ for a noise due to the following reasons: first, this noisy surface is a limit case in our study and the performance of our algorithm has to be tested, second, the recording devices could introduce white or pink noises that could influence the detection of the curvature radius, and thirdly, we could analyse the probability to detect a peak that does not exist. We then apply our algorithm and plot the result in Fig. B1. The following equation provides a good estimation of the fractal dimension $(\Delta=2.005)$ with coefficient of regression $r=0.99995$.

$\log r_{c}\left(l_{x}\right)=-0.914_{ \pm 0.006}+2.005_{ \pm 0.002} \log l_{x}+1.75_{ \pm 0.01} /\left(n_{x}-1\right)$

where $n_{x}$ is the number of $d_{x}$ intervals used to calculate $l_{x}$. Without applying the discretization errors that are particularly important in the case of a white noise, one would have obtained the following equation:

$\log r_{c}\left(l_{x}\right)=-0.4952+1.81 \log l_{x}$

with $\Delta=1.81$ and with a $20 \%$ error on the fractal dimension. Our modelling of the discretization error is then relatively efficient.
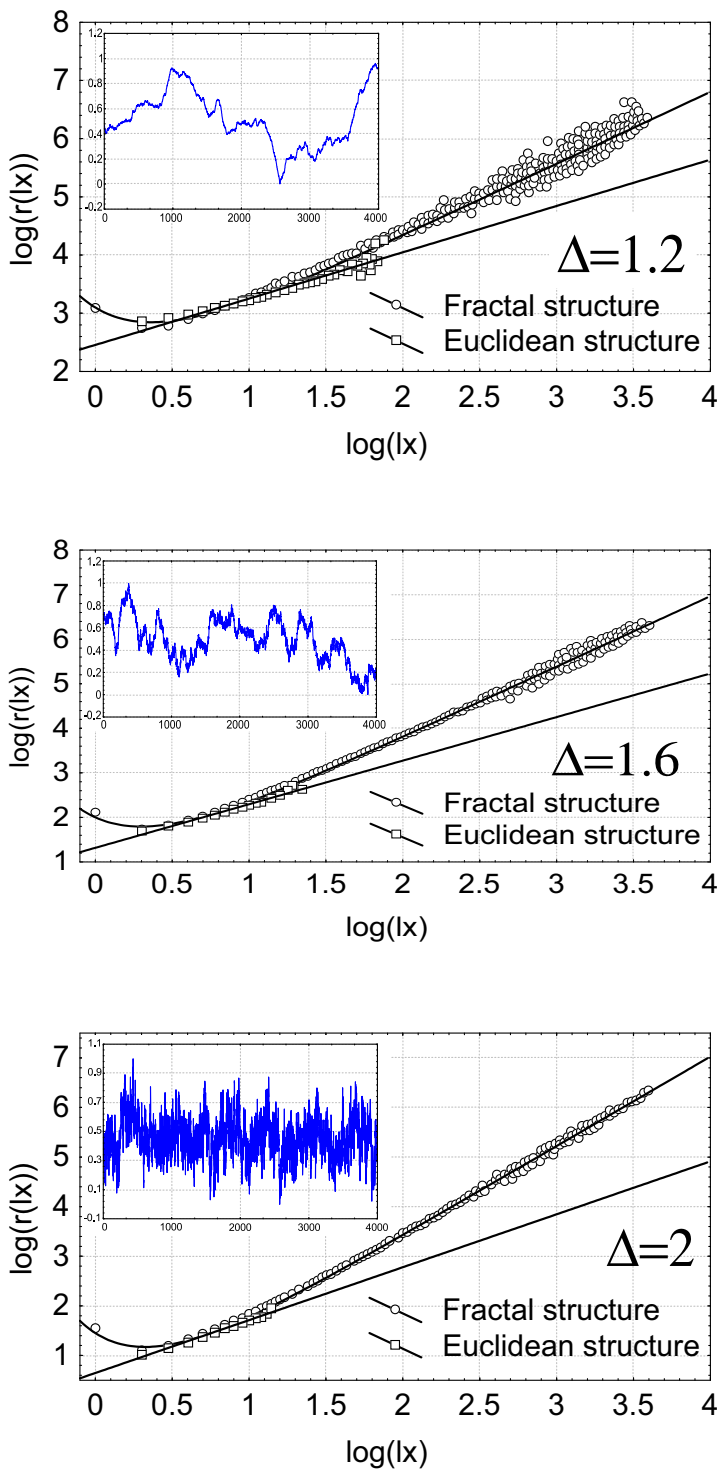

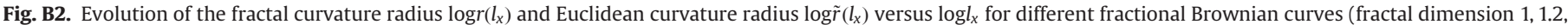
$1.4,1.6,1.8,2$ ) with their associated profiles. 


\section{The fractional Brownian motion}

The properties of a stochastic fractional Brownian motion (FBM) $[24,25]$ allow us to determine the algorithm effect on the determination of $r_{c}$ and $\tilde{r}$ for different fractal dimensions. The functions are determined by plotting different curves with different fractal dimensions ( 1 to 2 with 0.1 as discretization step). These curves are so discretized through 100,000 points. Since the FBM realize stochastic curves, 100 curves are then simulated for each fractal dimension. Fig. B2 shows the variation of $\log \tilde{r}_{c}\left(l_{x}\right)$ (Euclidian peaks, see Appendix A for mathematical definition) and $\log r_{c}\left(l_{x}\right)$ versus $\log l_{x}$ using the corrected method for $1,1.2,1.4,1.6$, 1.8 and 2 as theoretical fractal dimensions: first, Eq. (b1) fit very well the experimental data for any fractal dimension. The greater the fractal dimension is, the more pertinent is the corrected method. One obtains for the correction coefficient $\alpha_{2}, \alpha_{2}^{\Delta}=1=1.09 \alpha_{2}^{\Delta}=1.2=1.24, \alpha_{2}^{\Delta}=1.4=1.27$, and the following fractal dimensions $\Delta^{\Delta=1}=1.00, \Delta^{\Delta=1.2}=1.21, \Delta^{\Delta=1.4}=1.40$, $\Delta^{\Delta=1.6}=1.58, \Delta^{\Delta=1.8}=1.75$ and $\Delta^{\Delta=2}=1.92$. The fractal dimension estimated from the radius curvature of the surface is well found whatever the theoretical fractal dimension is. Those results are coherent, as far as, the number of peaks in the $l_{x}$ range decreases with the increase of the fractal dimension. For the Euclidean curve $(\Delta=1$, this curve is entirely differentiable), the regression lines of $\log \tilde{r}_{c}\left(l_{x}\right)$ and $\log r_{c}\left(l_{x}\right)$ versus $\log l_{x}$ are confounded. This means that both methods give the same estimation of the curvature radius for Euclidean curves. Since the fractal dimension increases, one obtains $\log \tilde{r}_{c}\left(l_{x}\right)<\log r_{c}\left(l_{x}\right)$ and the curvature radius is underestimated in the case of fractal curves, if we consider a peak as defined in Euclidian case (Appendix A). The difference increases with the fractal dimension confirming the hypothesis according to which, on the fractal curves, the peaks cannot be defined with the intuitive definition of the Euclidean curves.

\section{References}

[1] Mandelbrot BB, VAN NESS JW. Fractional Brownian motions, fractional noises and applications. SIAM Review 1968:422-37.

[2] Mandelbrot BB, WALLIS JR. Computer experiments with fractal Gaussian noises. Part 1, averages and variances. Water Resources Research 1969;10: 228-41.

[3] Paggi M, Ciavarella M. The coefficient of proportionality $k$ between real contact area and load, with new asperity models. Wear 2010;268:1020-9.

[4] Ciavarella M, Murolo C, Demelio G. On the elastic contact of rough surfaces: numerical experiments and comparison with recent theories. Wear 2006;261: 1101-13.

[5] Zavarise G, Borri-Brunetto M, Paggi M. On the resolution dependence of micromechanical contact models. Wear 2007;262:42-54.
[6] Wu J-J. The properties of asperities of real surfaces. ASME Journal of Tribology 2001:123:872-83.

[7] Ciulli E, Ferreira LA, Pugliese G, Tvares SMO. Rough contact between actual engineering surfaces. Wear 2008;264:1105-15.

[8] Nowicki B. Multiparameter representation of surface roughness. Wear 1985;102:161-76.

[9] Whitehouse DJ. In: Thomas TR, editor. Digital techniques, in rough surfaces; 1982. p. 144-66.

[10] Moalic H, Fitzpatrick JA, Torrance AA. The correlation of the characteristics of rough surfaces with their friction coefficients. Proceedings of the Institution of Mechanical Engineers 1987;201:321-9.

[11] Thomas TR. Recent advances in the measurement and analysis of surface microgeometry. wear 33 1975:205-33.

[12] Longuet-Higgins MS. Statistical properties of an isotropic random surface. Philosophical Transactions of the Royal Society of London, Series A 1957;250: 157-74.

[13] Whitehouse DJ, Archard JF. The properties of random surfaces of significance in their contact. Proceedings of the Royal Society of London Series A 1970;316:97-121.

[14] Sayles RS, Thomas TR. Measurements of the statistical microgeometry of engineering surfaces. Transactions on ASME, Journal of Lubrication Technology 1979; 101:409-18.

[15] Ganti S, Bhushan B. Generalized fractal analysis and its applications to engineering surfaces. Wear 1995;180:17-34.

[16] Gallant JC, Moore ID, Hutchinson MF, Gessler P. Estimating fractal dimension of profiles: a comparison of methods. Mathematical Geology 1994;26:455-81.

[17] Hall P. On the effect of measuring a self-similar process. SIAM Journal on Applied Mathematics 1995;55:800-8.

[18] Amada S, Yamada H. Introduction of fractal dimension to adhesive strength evaluation of plasma-sprayed coatings. Surface and Coatings Technology 1996;78(50-55):1996.

[19] Bozhelovny S, Vohnsen B. Fractal surface characterization: implications for plasmon polaritron scattering. Surface Science 1996;356:268-74.

[20] Doege E, Laackman B, Kischnick B. Characterisation of technical surfaces by means of fractal geometry. Steel Research 1995;66:113-6.

[21] Gagnepain JJ. Fractal approach to two-dimensional and three-dimensional surface roughness. Wear 1986;109:119-26.

[22] Tricot C. Curves and fractal dimension. Springer; 1995.

[23] Bech J, Bay N, Eriksen M. Entrapment and escape of liquid lubricant in metal forming. Wear 1999;232:134-9.

[24] Poon CY, Bhushan B. Comparison of surface roughness measurements by stylus profiler, AFM, and non-contact optical profiler. Wear 1995;190:76-88.

[25] Mc Cool JI. Comparison of models for the contact of rough surfaces. Wear 1896;107:37-60.

[26] Radhakrishnan V. Effect of stylus radius on the roughness values measured with tracing stylus instruments. Wear 1970;16:325-35.

[27] Whitehouse DJ. Theoretical analysis of stylus integration. Annals CIRP $1974 ; 23: 181-2$.

[28] Nakamura T. On deformation of surface roughness curves caused by finite radius of stylus tip and tilting of stylus holder arm. Bulletin of the Japan Society of Precision Engineering 1966;1(4):240-8.

[29] Bigerelle M, Iost A. 1996. Calcul de la dimension fractale d'un profil par la méthode des autocorrélations moyennées normées (AMN). Comptes Rendus de l' Academie des Sciences Paris, 323, Série IIb:669-675.

[30] Bigerelle M, Najjar D Iost A. Multiscale functional analysis of wear a fractal model of the grinding process. Wear 2005;258:232-9.

[31] Dubar L, Hubert C, Christiansen P, Bay N, Dubois A. Analysis of fluid lubrication mechanisms in metal forming at mesoscopic scale CIRP Annals-Manufacturing Technology 2012;61:271-4. 